\title{
El violinista José White en Estados Unidos: nueva información acerca de su gira norteamericana de 1875-1876
}

\author{
Violinist Joseph White in the United States: \\ New Light on his North American tour of 1875-1876
}

\author{
por \\ Yavet Boyadjiev \\ College of Music, Universidad Mahidol, Bangkok, Tailandia \\ yavetboyadjiev@gmail.com
}

José Silvestre de los Dolores White Laffita (1835-1918), también conocido como Joseph White, fue el primer violinista nacido en Cuba -y uno de los primeros en Latinoamérica- en alcanzar prestigio internacional y recibió algunas de las distinciones más importantes que su época podía ofrecerle. De ascendencia africana y francesa, y naturalizado francés, White llegó a tener una carrera internacional que le ganó un lugar entre los más respetados violinistas de su generación además de ser reconocido como uno de los ejemplos más prominentes de la Escuela Francesa de violín del siglo XIX. Hasta ahora, muy poco se ha conocido de su gira por Estados Unidos donde se convirtió en el primer solista de ascendencia africana en actuar con la orquesta que es ahora la New York Philharmonic. Mediante una descripción detallada de sus actividades en las ciudades de Nueva York, Boston, Brooklyn -entonces una ciudad independiente-, y Filadelfia, sobresalen las reacciones de la prensa, su círculo profesional, su estilo interpretativo y su repertorio, así como su contribución a causas que eran importantes para él.

Palabras clave: Cuba siglo XIX, Escuela francesa de violín, Theodore Thomas, Wieniawski, Carl Bergmann, New York Philharmonic, músicos de ascendencia africana, Joseph White, Ignacio Cervantes, Teresa Carreño, Emilio Agramonte.

José Silvestre de los Dolores White Laffita (1835-1918), also known as Joseph White, was the first Cuban-born violinist - and one of the first from Latin America as a whole- to achieve international stature and receive some of the most prestigious distinctions his period could offer. Of African and French heritage, and a French citizen by naturalization, White achieved an international career that placed him among the most respected violinists of his generation, and was recognized as one of the most prominent exponents of the French Violin School of the 19th

${ }^{1}$ Este trabajo es parte de un libro en preparación acerca de la vida y la obra de José White. Mis agradecimientos a Malena Kuss y a Allan Atlas por sus siempre sabias observaciones, a John Graziano por sus guía en asuntos histórico-musicales de Nueva York, a Kevin Schlottman y a Gabryel Smith de la New York Philharmonic por información pertinente a la orquesta, a Nicholas Sackman por sus valiosas aclaraciones del Stradivari Le Chant du Cygne, a Alexander Pérez-Heredia por sus sugerencias editoriales, y al comité editorial de $R M C h$ por los acertados comentarios críticos en esta investigación. 
century. Until now, little has been known about White's professional activities in the United States when, by forces of destiny, he became the first soloist of African descent -in any instrument- to perform with the orchestra that is now the New York Philharmonic. This article provides a detailed account of White's musical activities during this period: his concerts and professional collaborations, his repertoire, the reception he received from critics and audiences and his involvement in causes he held dear.

Keywords: 19th-century Cuba, French Violin School, Theodore Thomas, Wieniawski, Carl Bergmann, New York Philharmonic, African descent musicians, Joseph White, Ignacio Cervantes, Teresa Carreño, Emilio Agramonte.

\section{INTRODUCCIÓN}

El año 2018 marca el centenario del fallecimiento del violinista y compositor José Silvestre de los Dolores White Laffita (1835-1918), también conocido como José White o Joseph White. De ascendencia africana, White fue el primer violinista nacido en Cuba -y uno de los primeros nacidos en Latinoamérica-, en alcanzar estatura internacional, recibiendo algunas de las distinciones más prestigiosas que su época podía ofrecerle. Nacido en Matanzas durante la época colonial, su madre fue una joven liberta de origen africano -su apellido Laffita, españolización del apellido francés Laffitte, sugiere un pasado de esclavitud y un dueño francés-y su padre un comerciante de herencia francesa, nacido en la isla de Saint Domingue.

Con el apoyo de su familia White tuvo el privilegio de obtener una educación musical de calidad -la música era una de las pocas profesiones en las que los descendientes de africanos podían destacarse ${ }^{2}-$ y llegó a ser un compositor conocido de contradanzas y otras danzas de salón, director de orquesta para eventos sociales, compositor de música religiosa y violinista distinguido en los círculos musicales ilustres. En aquel entonces en Cuba no escaseaba la vida cultural y White fue capaz de absorber todo lo que pudo de su contexto nacional y de la cultura europea que llegaba a la isla por medio de los solistas y las compañías de ópera que con frecuencia añadían conciertos en La Habana y en Matanzas a sus itinerarios de giras en Norteamérica.

Durante su temprana juventud White se distinguió lo suficiente para que en 1854 le ofrecieran la oportunidad de un debut profesional acompañado por el pianista estadounidense Louis Moreau Gottschalk (1829-1869) ${ }^{3}$. Gottschalk parece haber sido una figura importante para que el joven White viajara a París a estudiar con el renombrado pedagogo Jean-Delphin Alard (1815-1888) ${ }^{4}$.

El primer éxito internacional de White ocurrió en París donde ganó el Primer Premio de violín del Conservatorio de París en 1856, a poco tiempo de haber iniciado estudios en esa institución. Como parte de ese premio, le otorgaron un violín Gand Frères (1856) especialmente hecho para él ${ }^{5}$. White, quien también fue reconocido en su tiempo como compositor y como pedagogo, llegó a convertirse en uno de los más sobresalientes exponentes de la Escuela Francesa del violín, y en uno de los pocos músicos de ascendencia africana que contribuyeron al desarrollo de la música académica europea del siglo XIX y a la expansión de la cultura europea fuera de ese continente.

2 Boyadjiev 2015: 15.

3 Toledo 1990: 12, y Giro 2007: 287.

4 Trotter 1878: 225. La idea de que Gottschalk haya alentado al joven White a estudiar en París reaparece con frecuencia en la literatura sobre White.

5 Este violín lleva el nombre "Joseph White" y la fecha del premio inscritos en pan de oro a lo largo de los aros. Para imágenes y documentación del violín Gand Frères (1856) ver: Boyadjiev 2015: 20; y Boyadjiev 2018: 46. 
A pesar de haber nacido en Cuba y adoptado en 1870 la ciudadanía francesa ${ }^{6}$, White fue un ciudadano del mundo en el mismo sentido que podríamos definir este término en la actualidad. Fue también un emigrante y artista que desarrolló su carrera como parte de la pequeña diáspora de músicos cubanos que fueron a Europa para mejorar sus vidas, no solo por haber sido expulsado de Cuba en 1875 por razones políticas. Fue también porque únicamente fuera de la isla, en lugares que ofrecieran mejores oportunidades para el reconocimiento de su talento y menos limitaciones sociales para los mestizos y descendientes de africanos, podría él aspirar a lograr sus más altas metas profesionales, además de mejorar el nivel de vida de su familia.

White fue también el dueño del violín Stradivari de 1737, Le Chant du Cygne, que adquirió en $1887^{7}$. Este violín tiene la distinción histórica de ser considerado el último fabricado por el luthier cremonense.

La estatura artística de White fue reconocida durante su vida por notables de la música como Gounod, Rossini, Saint Saëns y Fauré, entre otros. Fue merecedor de premios honoríficos y títulos de la República Francesa, del presidente de Venezuela Francisco Linares Alcántara, y de miembros de casas reales, incluyendo a la reina Isabel II y el rey Alfonso XII (España), el emperador Dom Pedro II (Brasil), el rey Carol I (Rumanía), y el papa León XIII, quienes lo colmaron de honores.

Excluyendo a Cuba, a Francia y a Brasil, los tres países donde él vivió por largos períodos, White realizó giras de conciertos en varios países latinoamericanos, España, Mónaco, Inglaterra y Estados Unidos. La Revista Musical Chilena, en su número XLIV/173 (enerojunio, 1990), parcialmente dedicado a White, reportó ampliamente acerca de su extensa gira de 1877 a 1879 por América Latina ${ }^{8}$.

Hasta la fecha, las actividades de White en Estados Unidos no han sido propiamente documentadas más allá de unas pocas referencias a conciertos específicos en Nueva York y en Boston. El propósito de este artículo es documentar en detalle y de manera mayormente cronológica sus actuaciones en ciudades de la costa este de Estados Unidos, además de destacar la importancia de algunas de estas presentaciones tanto para la comunidad cubana que se encontraba en Estados Unidos como para la historia de algunas de las más importantes instituciones musicales norteamericanas del siglo XIX.

Para documentar esta etapa profesional de White he trabajado con publicaciones norteamericanas periódicas y de otra índole. De especial interés son las críticas especializadas en Dwight's Journal of Music (1852-1881), diario importante para la historia de la crítica musical en Estados Unidos, y el perfil biográfico de White escrito por James M. Trotter en Music and Some Highly Musical People (1878), fuente indispensable para el conocimiento de las actividades de los músicos de ascendencia africana antes y después de la Guerra Civil -Trotter además conoció a White y lo vio actuar en concierto-. A estos se suman libros escritos por personas con quienes White se relacionó, además de prensa europea, registros en archivos nacionales de Estados Unidos y de Francia, y programas de conciertos preservados

6 Boyadjiev 2015: 38 y 50; D’Arcier 1997: 104-105.

7 White compró el violín Le Chant du Cygne en 1887. En previas publicaciones mías (2018 y 2015) la fecha aparece como 1886 pero esto es un error. Nicholas Sackman, quien generosamente compartió conmigo el registro de ventas de este instrumento, merece todo el crédito por esta corrección. Información en The Jacques Français Rare Violins Inc. Photographic Archive and Business Records, The sales ledgers, 1845-1938, Smithsonian Institution.

8 Para información sobre la gira de White en Venezuela, ver: Milanca Guzmán 1990b: 25-64. Para información sobre la visita de White mayormente en Chile además de información sobre sus actividades en Perú, Uruguay, Panamá y Argentina, ver: Merino 1990: 65-113. 
en los archivos de Brooklyn Historical Society, Historical Society of Pennsylvania y New York Philharmonic Leon Levy Digital Archives.

Las actividades de White en las ciudades de la costa este de Estados Unidos fueron parte de una gira de once meses, durante estos, sus actuaciones estuvieron concentradas en un período de nueve meses. La gira incluyó las ciudades de Nueva York, Brooklyn -hasta 1898 ciudad independiente-, Boston y Filadelfia ${ }^{9}$, con presentaciones en algunas de las salas más importantes del país y con auspicio de algunas de las instituciones musicales y directores de más renombre. Dispersas y limitadas menciones sobre esta gira han aparecido en la literatura previa de White ${ }^{10}$.

\section{PRIMEROS CONCIERTOS EN NUEVA YORK Y BROOKLYN}

Aunque ha sido difícil encontrar la fecha exacta del arribo de White a Nueva York, se puede afirmar con certeza, por una nota en The New York Times, que tanto él como su amigo Ignacio Cervantes (1847-1905) ya se encontraban allí el 15 de agosto de $1875^{11}$. También del 15 de agosto es esta nota del New York Herald que informa al público acerca de los próximos conciertos:

"Mr. José White, violinista cubano, alumno de Alard y uno de los más eminentes alumnos del Conservatoire de Paris, ha llegado a Nueva York y propone ofrecer una serie de conciertos aquí el próximo mes. Valmaseda lo exilió de Cuba, celoso de su popularidad como artista, y sus últimas actividades profesionales en México le han agregado considerablemente a su renombre europeo. En París, Mr. White es considerado segundo solo con respecto a Alard, a cuyos alumnos él ha instruido durante la ausencia del maestro. Fue condecorado por la Reina de España, disfrutó de la amistad del Emperador Napoleón (Ay!) y actuó en las veladas de Rossini”12.

Eran tiempos difíciles entre Cuba y España. La guerra de liberación que había comenzado en 1868 y que duraría diez años estaba en su apogeo. White y Cervantes habían sido desterrados ese mismo año después de una presentación en el Teatro Tacón de La Habana en que se escucharon gritos patrióticos desde la audiencia ${ }^{13}$. La noticia de la expulsión de White curiosamente había sido reportada por el New Orleans Republican y The Philadelphia Inquirer, reproducida aquí como aparece en este último:

9 D'Arcier 1997:144 menciona que White actuó en Cincinnati, de lo que no existe evidencia alguna.

10 Trotter 1878: 224-240 ofrece un perfil biográfico de White que incluye información parcial respecto de esta gira mencionando dos conciertos en 1876: el 12 de marzo en Nueva York y el 26 de marzo en Boston, este último concierto presenciado por él. D’Arcier 1997:142-144 y Trujillo 2005: 58-59 hacen mención general a conciertos de White e Ignacio Cervantes en Nueva York, sin mencionar fechas. D'Arcier 1997:144 menciona un presunto concierto en esta ciudad, dirigido por Theodore Thomas el 20 de marzo de 1876, pero este es un dato erróneo.

11 “Approaching Concerts", The New York Times, XXIV/7461 (15 de agosto, 1875): 6.

12 New York Herald, XXXV/14237 (15 de agosto, 1875): 7. Blas de Villate y Hera, conde de Valmaseda (1824-1882), militar español, era el capitán general de Cuba en 1875. Esta cita y todas las siguientes cuya versión original está en inglés han sido traducidas al castellano por la autora. Se ha mantenido la ortografía original dentro de lo posible, incluyendo los nombres de las obras musicales tal cual aparecen deletreadas en la redacción original de la publicación. Sin embargo, en el texto principal de este artículo se alude a dichas obras utilizando el nombre dado originalmente por el compositor.

13 Toledo 1990: 19. 
"CUBA. Un músico mulato desterrado. Habana, 7 de mayo". -El jefe de la policía le ha notificado hoy a Jose [sic] White, un violinista de celebridad local y graduado del Conservatorio de París, que por orden del gobierno debe abandonar la isla. Senor[sic] White es un mulato nativo de Matanzas, pero dice ser ciudadano francés. Él ha profesado completa ignorancia sobre la causa de su destierro ${ }^{14}$.

Después de estos sorpresivos eventos, el 11 de mayo ${ }^{15}$ White salió de Cuba hacia México donde ofreció conciertos y también viajó por corto tiempo a París, antes de reunirse con Cervantes en Nueva York. Una vez allí, los primeros anuncios de prensa concernientes al violinista fueron de carácter biográfico y el objetivo era darlo a conocer al público norteamericano para el que él era completamente desconocido. Es evidente que toda la información biográfica contenida en esas notas introductorias le fue proporcionada a la prensa por el mismo White, quien viajaba con la documentación de respaldo necesaria.

White y Cervantes ofrecieron tres conciertos en Steinway Hall, entonces en 14th Street, en Manhattan, el 19, el 23 y el 26 de octubre de 1875, los que fueron muy anunciados en The New York Times ${ }^{16}$. Steinway Hall fue, hasta la inauguración del Carnegie Hall en 1890, la sala más importante de la ciudad, capaz de alojar en su sala principal a una orquesta sinfónica completa ${ }^{17}$. Nueva York en ese momento hospedaba a una gran cantidad de cubanos expatriados, los que luchaban una guerra propagandística bajo la organización del Comité Revolucionario Cubano de esa ciudad, en apoyo al movimiento independentista cubano de la Guerra de los Diez Años. Este pequeño pero influyente grupo constituyó la gran parte de la audiencia de White durante sus primeros recitales en la ciudad, y parte de las ganancias de estos conciertos seguramente se destinaron como donaciones para la causa independentista de Cuba. La asociación de White y Cervantes con el músico cubano Emilio Agramonte (1844-1918) y otros, pudo haber resultado en otros conciertos privados (no anunciados) para recaudar fondos para apoyar a los rebeldes en la isla ${ }^{18}$. La congregación de tantos coterráneos en estos conciertos no pasó inadvertida para el reportero de The New York Times, quien tomó notas de las efusivas expresiones en español que provenían de la audiencia:

"Conciertos White-Cervantes". Messrs. White y Cervantes, el primero, un violinista educado en París y muy respetado en esa ciudad y, el segundo, un pianista cubano, dieron anoche el primero de dos conciertos -el segundo ocurrirá mañana- en Steinway Hall. Ambos caballeros, si no nos equivocamos, son exiliados de la siempre-fiel isla debido a sus opiniones políticas y por lo tanto el gran entusiasmo con que su presentación fue

14 "Cuba. A mulatto musician banished. Havana, May 7", The Philadelphia Inquirer, XLVI/s/n, (8 de mayo, 1875): 1; "Ordered to leave. Havana. May 8”, New Orleans Republican, IX/26 (9 de mayo, 1875): 8.

15 "Cuba", The York Daily (Pennsylvania), XIV/1370 (12 de mayo, 1875): 4.

16 "Steinway Hall White-Cervantes", The New York Times, XXV/7509 (10 de octubre, 1875): 11. "Steinway Hall White-Cervantes", The New York Times, XXV/7519 (21 de octubre, 1875): 7; "Steinway Hall White-Cervantes", The New York Times, XXV/7520 (22 de octubre, 1875): 9. "Steinway Hall WhiteCervantes Grand Concert", The New York Times, XXV/7519 (21 de octubre de 1875): 7; "Steinway Hall White-Cervantes Grand Concert", The New York Times, XXV/7520 (22 de octubre, 1875): 9.

17 Hoover 2001; Slonimsky 1958: 1566; Steinway Hall 2016.

18 Emilio Agramonte, original de Camagüey, fue maestro de piano y de voz en Nueva York. Junto a otros músicos cubanos que apoyaban la causa cubana -entre ellos la cantante Amalia Simoni (1842-1918), viuda del general cubano Ignacio Agramonte (1841-1873)- él formó un grupo muy comprometido con el movimiento patriótico de Cuba y organizaban conciertos benéficos y recaudaban fondos de ayuda a los insurgentes cubanos en la isla (Lapique 2008: 216). 
recibida por la representación de Cuba Libre fue sin duda ampliamente [justificado]. Gran parte del aplauso, sin embargo, fue merecido. Mr. White, un alumno de Alard, nos decepcionó un poco en la fantasía sobre temas de "Robert" de su maestro, ya que en la interpretación se observó una falta de libertad y de amplitud en pasajes difíciles. En la pequeña pieza con la que él respondió a un encore Mr. White tuvo más éxito, su tono era puro y redondo y su [uso del] arco bueno. Más tarde, en la "Chaconne" de Bach, él mostró una maestría técnica que no había sido aparente al principio. [... $]^{19}$.

Coincidiendo con el reportero de The New York Times, New York Tribune también consideró que la interpretación de White de la Ciaccona de Bach fue el momento culminante de la noche. La mayoría de las palabras aduladoras fueron dirigidas a White, no tanto a Cervantes:

"Música. Conciertos White-Cervantes". En la ocasión del debut de anoche de los Señores White y Cervantes, artistas cubanos, Steinway Hall estuvo casi llena con una audiencia compuesta mayormente por sus compatriotas, quienes le dieron una cálida recepción y exhibieron un deseo de repetir casi indiscriminadamente casi todas las piezas [que formaban parte] del programa. El Señor White seleccionó una fantasía sobre temas de "Robert le Diable" de Alard, una Chaconna de Bach y su propia pieza de concierto "La Styrienne". En la fantasía, que fue su pieza inicial, él produjo una impresión favorable. Su técnica es admirable, tiene gran amplitud de estilo y su sonido es redondo y generoso e inusualmente puro. [...] Respecto al señor Cervantes -aunque quizás de más popularidad- el éxito no fue tan completo en el sentido artístico como en el caso de su colega $[\ldots]^{20}$.

Después del último concierto de la serie, el New-York Tribune publicó una crítica en términos más efusivos que la primera, esta vez comparando a White con el famoso virtuoso Henryk Wieniawski (1835-1889)21, con palabras aún más favorables hacia White. Wieniawski había realizado una gira de conciertos en Estados Unidos desde 1872 hasta $1874^{22}$, y sus actuaciones todavía formaban parte de la memoria cultural de las ciudades que él visitó. De hecho, esta es la primera de varias reseñas de diversas fuentes en las que se compara a White con Wieniawski durante esta gira:

"Música. Conciertos White-Cervantes". La serie de conciertos ofrecidos por los señores White y Cervantes ha sido la manera de presentarle al público a nuestros dos artistas, ambos de habilidades excelentes y uno de ellos en cierta medida el mejor ejecutante de su especial instrumento que ha visitado a América en los últimos años. El Señor Josef[sic] White, quien llegó aquí sin promoción alguna, es graduado del Conservatoire de Paris, alumno de Alard, y ha sido un gran favorito en París durante algunos años. Fresco de las salas de París, unas de las más serias en cuanto a los virtuosos del mundo, su trabajo ha sido asociado, en todos los sentidos, con un muy alto estándar de excelencia artística y todavía no ha tenido tiempo de sufrir el deterioro artístico que parece originarse al actuar ante las audiencias americanas -al cual ni el gran artista Wieniawski ha podido escapar completamente- [...]. Señor White parece tener una meta más alta

19 "The White-Cervantes Concerts", The New York Times, XXV/7518 (20 de octubre, 1875): 4.

20 "Music. White-Cervantes Concerts", New-York Tribune, XXXV/10781 (20 de octubre, 1875): 5.

21 Schwarz y Chechlińska 2001.

22 La gira de Wieniawski en Estados Unidos desde 1872 hasta 1874 fue cubierta en detalle por la prensa local de las ciudades que visitó. 
que esta [última], su esfuerzo ha sido el no presentar nada que no fuera admirable desde el punto de vista técnico pero también desde el punto de vista artístico. [... $]^{23}$.

Con el éxito de audiencia y de crítica de la serie White-Cervantes Concerts, el nombre de White se estableció en Nueva York como el de un solista internacional por quien valía la pena pagar para ir a ver. La frecuencia de sus presentaciones públicas se incrementó e incluyó no solo conciertos de alto perfil sino también otros de carácter más modesto, como los conciertos benéficos o presentaciones en iglesias durante los servicios. A veces la prensa lo anunciaba como cubano y otras como francés o "violinista parisino", como ocurrió en un anuncio para una presentación el 7 de noviembre en Saint Peter's Church, de Hick Street ${ }^{24}$.

Su primera presentación en la sala de Brooklyn Academy of Music, situada en Montague Street, Brooklyn Heights ${ }^{25}$, ocurrió el 22 de noviembre de 1875, en un concierto benéfico titulado "Grand Musical Soirée". El concierto fue para el beneficio de la Sheltering Arms Nursery, una organización cristiana de ayuda a niños vulnerables fundada en $1870^{26}$. El elenco incluyó a la reconocida cantante Emma Thursby (1845-1931) quien había ya colaborado durante la serie White-Cervantes en Steinway Hall, y el grupo de cantantes The Young Apollo Club. El pianista fue George Colby y las piezas del programa fueron la Fantaisie sur "Martha" de White, y la Grande Fantaisie sur "Robert le Diable" de Alard27. Al día siguiente The Brooklyn Daily Eagle publicó una reseña en la que White fue comparado otra vez con Wieniawski:

"Sheltering Arms Soirée Musicale" [...] [White] es sin duda el mejor intérprete del violín, con la excepción de Wieniawski, a quien se le ha escuchado aquí en años, y de alguna forma él es aún superior $[\ldots]^{28}$.

Sin embargo, sería el próximo concierto el que constituiría un hito en la carrera de White, cuando fue llamado a interpretar como solista el Concierto para violín en mi menor, op. 64, de Félix Mendelssohn-Bartholdy con la Philharmonic Society of New York (actualmente la New York Philharmonic), bajo la dirección de Carl Bergmann. Quizás sin saberlo o quizás estando acostumbrado a ser parte de este tipo de estadística, White se convirtió en el primer solista de ascendencia africana en actuar con la Philharmonic Society of New York ${ }^{29}$, la orquesta de más antigüedad en Estados Unidos (ver Figura 1) ${ }^{30}$.

La excepcionalidad de este hecho merece unas líneas adicionales para que se entienda mejor. Desde la fundación de la orquesta, en 1842, ningún otro solista o miembro

23 "White-Cervantes Concerts", New-York Tribune, XXXV/10787 (27 de octubre, 1875): 4-5.

24 "Father Fransioli", The Brooklyn Daily Eagle, XXXVI/263 (5 de noviembre, 1875): 3.

25 La actual Brooklyn Academy of Music está situada, desde 1903, en Lafayette Avenue, Brooklyn.

26 Brooklyn Historical Society 2018.

27 "Academy of Music, Brooklyn", The Brooklyn Daily Eagle, XXXVI/276 (20 de noviembre, 1875): 1; "Sheltering Arms Nursery", The Brooklyn Daily Eagle, XXXVI/277 (22 de noviembre, 1875): 3.

28 "Sheltering Arms Soirée Musicale", The Brooklyn Daily Eagle, XXXVI/278 (23 de noviembre, 1875): 3 .

29 Información confirmada por Gabryel Smith, Assistant Archivist, New York Philharmonic (11 de enero, 2018).

30 La orquesta que ahora conocemos como New York Philharmonic fue fundada en 1842 con el nombre de Philharmonic Society of New York y es la orquesta de más antigüedad en Estados Unidos. Desde 1928 se unió a la New York Symphony Orchestra para formar desde ese año la que es actualmente conocida como Philharmonic-Symphony Society of New York, Inc. o New York Philharmonic (Haws 2013). 


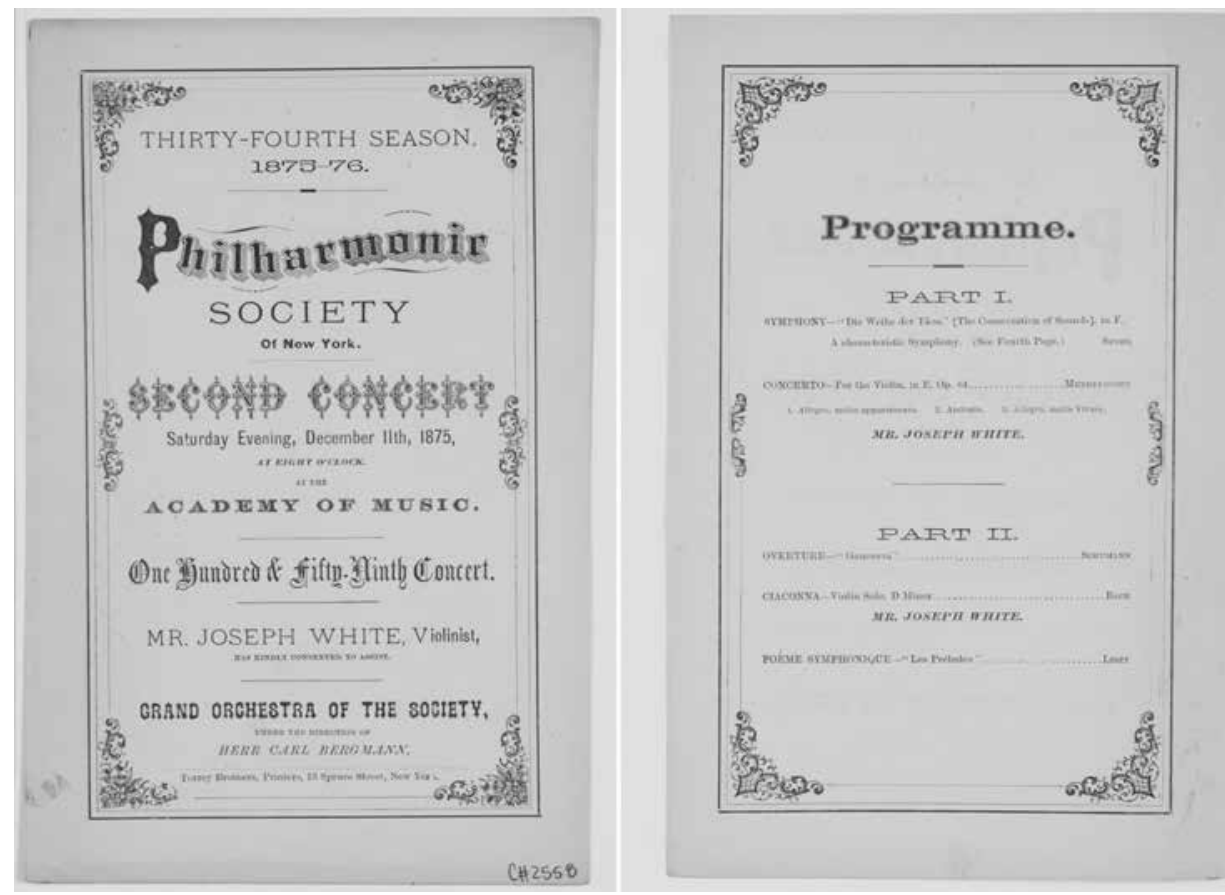

Figura 1. Programa del debut de White con la Philharmonic Society of New York, ahora New York Philharmonic, el 11 de diciembre de 1875. Academy of Music, New York. Cortesía de New York Philharmonic Leon Levy Digital Archives, Program no. 2558.

de la orquesta había sido de ascendencia africana hasta que White actuó como solista en 1875. Estados Unidos era un país con una sociedad segregada, a solo diez años de la abolición oficial de la esclavitud y del fin de la Guerra Civil (1861-1865), por lo que para los descendientes de africanos, que eran mayoritariamente descendientes de esclavos como también lo era White, las oportunidades profesionales eran muy limitadas, especialmente en el contexto de la tradición musical europea, donde además eran una escasa minoría. Sin embargo, es importante hacer una distinción entre la manera en que las audiencias y los críticos percibían a un músico de ascendencia africana nacido en Estados Unidos y a otro nacido en otro país. El que White fuera cubano de nacimiento y francés por naturalización le daba la ventaja de ser percibido como "exótico" y esto sin duda contribuyó a sus oportunidades profesionales en Estados Unidos. Por esta razón es justo aclarar que, aunque White pudo haber experimentado algún tipo de discriminación racial, su posición privilegiada como "extranjero" atenuó las consecuencias que hubiera podido sufrir por su ascendencia africana. Además del aspecto racial, hay suficiente evidencia para afirmar que, en esa época, los solistas europeos eran recibidos con los brazos abiertos mientras que a los estadounidenses, aunque hubieran alcanzado éxitos en Europa, les era mucho más difícil obtener reconocimiento en su propio país ${ }^{31}$.

31 Gottschalk (2006: 46) expresa con frustración al volver a actuar en Estados Unidos: “[mi] reputación no había, de alguna manera, cruzado el Atlántico”. Lott (2003: 48, 109) trata el tema de la preferencia del público norteamericano por los solistas extranjeros. 
Si treinta y tres años habían transcurrido para que un "extranjero" de ascendencia africana se presentara como solista, cincuenta años más tendrían que transcurrir para que un estadounidense descendiente de africanos pudiera hacerlo, en 1925, y aún treinta y tres años más tendrían que transcurrir para que, en 1962, un estadounidense descendiente de africanos fuera aceptado como miembro permanente de la orquesta ${ }^{32}$.

El debut de White con la Philharmonic Society of New York en la Academy of Music, Manhattan, fue reseñado en The New York Times y en Dwight's Journal of Music, ambos en términos muy favorables. El crítico de The New York Times comparó la interpretación de White con la de Wieniawski -este último había interpretado el mismo concierto de Mendelssohn con la Philharmonic durante la temporada 1873-1874-, proclamando que la interpretación de White de la Ciaccona de Bach nunca había sido igualada. La reseña en The New York Times describe el debut de White como un éxito rotundo:

"La Philharmonic anoche". El concierto de ayer en la Academy of Music [...] El incidente más interesante del concierto fue la interpretación al violín del Señor Joseph White. Un tono más puro, un fraseo más delicado y una entonación más pura que las que caracterizan a este joven artista, nunca han sido disfrutadas por el público local. Posiblemente, la ejecución del concerto de Mendelssohn de M. Wieniawski tenía un poco más de brío, pero no estamos seguros ahora y en la exactitud de las doble cuerdas, la obra del más viejo intérprete no sufrió ni un poco por este maravilloso brío. La interpretación del Señor White fue simplemente perfecta, el bello andante de la composición reveló la dulzura y expresión del cantabile. Fuertes aplausos siguieron a esta parte del concierto, pero una inusual demostración de placer respondió a la interpretación del Señor White de la Ciaconna de Bach, después de haber sido recibido con vítores en su segunda aparición en el escenario. La interpretación de esta pieza por este violinista nunca ha sido igualada aquí y el tributo, en el que la orquesta se unió a la audiencia, fue completamente merecido ${ }^{33}$.

La autoridad de la crítica musical del momento y quizás una de las publicaciones que más influenció el desarrollo de la crítica musical en Estados Unidos en el siglo XIX, Dwight's Journal of Music, consideró la interpretación de White del más alto calibre. De acuerdo con el corresponsal de Dwight's en Nueva York:

Totalmente deliciosa e inspiradora fue la ejecución en violín del solista, Mr. Joseph White, quien interpretó el Concerto en Mi [menor] de Mendelssohn, y la Ciaconna en Re menor de Bach. Todas las partes del bello concierto fueron interpretadas lo mejor posible, yo he escuchado pocas veces una interpretación tan satisfactoria, mientras

32 Los años mencionados se han recopilado de Shanet 1975: 347, y de Gabryel Smith, Archivero Asistente de la New York Philharmonic (en comunicación por correo electrónico del 4 de julio, 2018). Smith confirmó que "White fue el único solista con raíces africanas hasta 1925 cuando Marian Anderson y Roland Hayes actuaron en la misma temporada". Shanet no es muy concluyente cuando llama a White "uno de los primeros solistas negros en trabajar con la Philharmonic", sin embargo, no menciona ningún otro nombre además del de White. Esto en sí es significativo, pues Shanet, para escribir este libro, tuvo acceso a la gran mayoría de registros históricos de la New York Philharmonic. Shanet, sin embargo, es categórico en cuanto a que en 1962 el violinista estadounidense Sanford Allen se convirtió en el primer músico descendiente de africanos en ser miembro a tiempo completo de la orquesta.

33 "Last Evening's Philharmonic", The New York Times, XXV/7563 (12 de diciembre, 1875): 6. 
que la Ciaconna, que demanda talento y cualidades artísticas del más alto orden, fue igualmente bien interpretada ${ }^{34}$.

Y éxito en la prensa significaba prácticamente todo, pues la prensa podía hacer o destruir la carrera de un solista, y en el caso de White este éxito significó que recibiera una segunda invitación para actuar como solista con la orquesta tres meses más tarde ${ }^{35}$.

Después de este debut como solista, White formó parte de un concierto de música de cámara con la joven y ya afamada pianista venezolana Teresa Carreño (1853-1917), quien tenía veintiún años de edad en ese momento y con su esposo -con quien se había casado hacía dos años, en 1873- Émile Sauret (1852-1920), de entonces veinticinco años de edad. El concierto tuvo lugar en el entonces auditorio de conciertos -ahora inexistenteChickering Hall, en Manhattan, el 17 de diciembre. Ignacio Cervantes y el cellista Charles Werner fueron también parte de este evento. Carreño y Sauret estaban en Estados Unidos formando parte de una troupe musical liderada por la soprano Ilma di Mirska (1836-1889) ${ }^{36}$.

El programa incluyó el Cuarteto en si menor, op. 3, de Mendelssohn -White ejecutando la viola-, solos de Nardini y de Spohr interpretados por Sauret, una Ballade de Chopin interpretada por Cervantes, un dúo de Schumann para dos pianos por el dúo CarreñoCervantes, y la Sonata op. 30, no. 3, de Beethoven, por el dúo White-Carreño ${ }^{37}$. La reseña que apareció en el New-York Tribune, aunque crítica sobre las interpretaciones de Sauret, destaca positivamente la colaboración White-Carreño y en especial la ejecución de White:

“Conciertos Clásicos y Populares”. Una asociación de artistas, comprendida por Mme. Carreno[sic]-Sauret, Messrs. Cervantes, White, Sauret and Werner, ofrecieron anoche el primer concierto de una serie en Chickering Hall [...] [El concierto] abrió con el Cuarteto en Si menor de Mendelssohn para piano e instrumentos de cuerdas, con Mme. Carreno[sic]-Sauret al piano, Messrs. Sauret al violín, White en la viola y Werner en el cello. El Allegro estuvo un poco demasiado lento y fue un poco pesado; el Andante sin embargo estuvo mucho mejor, fue interpretado suavemente con mucho sentimiento, y fue solo dañado por alguna nota ocasionalmente desafinada en el violín. El Scherzo casi nunca fue delicado y el sonido de Mr. Sauret no muy elocuente, así que con frecuencia su parte no se escuchaba. El mismo problema ocurrió con el Finale. [...] Mme. Carreno[sic]-Sauret y Mr. White tocaron la Sonata en Sol mayor, op. 30, no. 3 de Beethoven para piano y violín y esta fue quizás la interpretación que más disfruté. Las partes estuvieron bien balanceadas y ambos intérpretes estuvieron en su mejor forma. $[\ldots]^{38}$.

34 Dwight's Journal of Music, XXXV/20 (8 de enero, 1876): 159.

35 Shanet 1875: s/n, Figura 23 (subtítulo de imagen sin número de página) "José (Joseph) White, violinista Negro nacido en Cuba, actuó con la Philharmonic en 1875-76 con semejante éxito que fue invitado para un segundo concierto en la misma temporada".

36 Stevenson 1983: 9-15. Para más información de Teresa Carreño y sus giras ver: Stevenson 2004:163-179. Para un sumario de su vida y su obra ver "6. Carreño García de Sena, Teresa”, Milanca Guzmán 2002: 234-237. Acerca de la relación de amistad entre Carreño y White ver Milanca Guzmán 1990: 69-83.

37 Dwight's Journal of Music, XXXV/20 (8 de enero, 1876): 159.

38 "Popular and Classical Concerts", New-York Tribune, XXXV/10832 (18 de diciembre, 1875): 7. 


\section{DEBUT EN FILADELFIA Y DE VUELTA A NUEVA YORK Y A BROOKLYN}

White abrió el nuevo año, con cuarenta y un años de edad (cumplidos el 31 de diciembre de 1875), debutando como solista en Filadelfia y repitiendo tanto el concierto de Mendelssohn como la Ciaccona de Bach, con The Thomas Orchestra y Theodore Thomas (1835-1905) a la batuta. El concierto tuvo lugar en la Academy of Music en Filadelfia, el 13 de enero de 1876 (ver Figura 2):

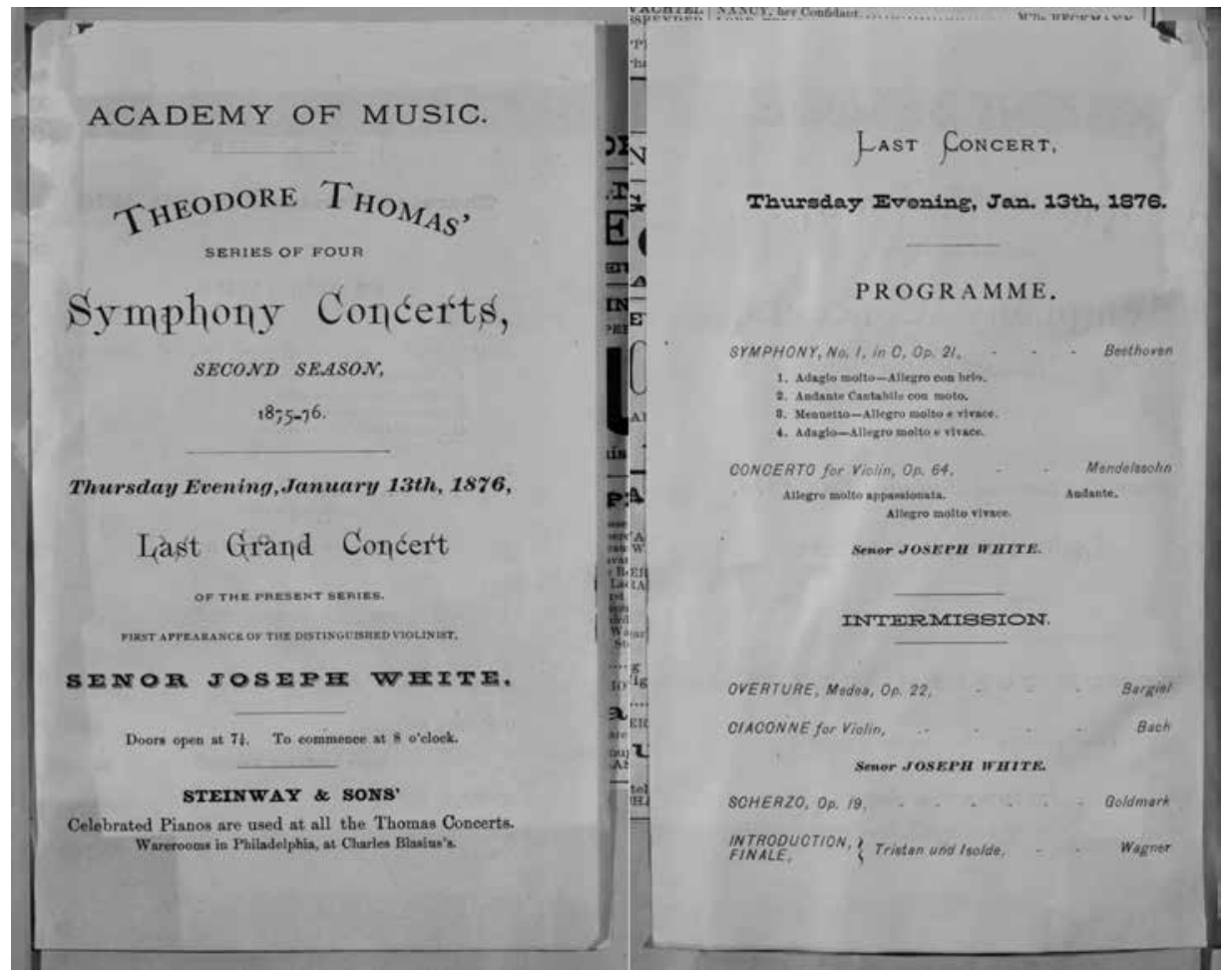

Figura 2. Programa del debut de White en Philadelphia, el 13 de enero de 1876, con The Thomas Orchestra. Academy of Music, Philadelphia. Historical Society of Pennsylvania, Scrapbook Vol. 16, 1873-1876.

The Thomas Orchestra era considerada una de las mejores -quizás la mejor- de la época, y tenía la ventaja de tener un calendario de ensayos a tiempo completo, por lo que su resultado artístico era con frecuencia de mejor calidad que el de su homóloga, la Philharmonic Society of New York ${ }^{39}$. Aunque estas dos orquestas eran vistas en relación de competencia, la realidad era que compartían muchos de los músicos, directores y solistas. De hecho, varios solistas que actuaban con la Philharmonic también actuaban como solistas 
con la orquesta de Theodore Thomas -a veces en la misma temporada-como es el caso de White, Wieniawski, Sarasate y A. Rubinstein, por citar solo algunos ${ }^{40}$.

Este debut de White en Filadelfia fue reseñado brevemente en el periódico local The Times y es hasta ahora el único ejemplo encontrado en la prensa norteamericana durante la gira de White, donde el corresponsal, aunque comenta favorablemente acerca de la calidad de su interpretación, también se refiere a la apariencia física del violinista y a su color de piel. Durante toda la gira en Estados Unidos la apariencia física de White no parece haber sido motivo de atención en la mayoría de los reportes de prensa encontrados.

"Los Conciertos Sinfónicos de Thomas". El último en la serie de conciertos sinfónicos fue ofrecido anoche en la Academy of Music por la Orquesta de Theodore Thomas. [...] La novedad del evento fueron los solos del Senor[sic] White, un cubano que es suficientemente oscuro como para tener sangre africana en sus venas. Él es un artista consumado y toca con acabado y elegancia clásicos, aunque su estilo no está marcado por gran amplitud o fervor. [White] interpretó el familiar concerto de Mendelssohn y la Ciaconne de Bach para violín. Ambas obras fueron aplaudidas con entusiasmo y la última recibió un encore $[\ldots]^{41}$.

Después de su debut en Filadelfia, White y Thomas se trasladaron a Brooklyn donde dos días más tarde, el 15 de enero, repitieron el concierto de Mendelssohn, esta vez con la Philharmonic Society of Brooklyn ${ }^{42}$ en la Brooklyn Academy of Music. Además de White, la cantante Emma Thursby participó como solista ${ }^{43}$. Los organizadores le permitieron a la prensa y a miembros de la audiencia disfrutar de un ensayo abierto la noche antes del evento, y The Brooklyn Daily Eagle reportó pertinente a la experiencia de ambos días ${ }^{44}$, de nuevo comparando a White con Wieniawski en la reseña del día del concierto:

"Segundo Concierto de la Philharmonic". En el grupo de los conciertos notables ofrecidos por la Philharmonic Society, el de la noche del sábado -el segundo concierto de la decimoctava temporada-merece formar parte, y posiblemente colocarse de primero, en virtud de su excepcional excelencia. [...] Mucho entusiasmo contenido se liberó [esa noche] en reconocimiento a la magnífica interpretación del Signor Josef[sic] White [...] De la interpretación del Senor[sic] White ya hemos hablado en los más altos términos de encomio. Desde [la visita de] Wieniawski, ningún otro violinista que ha visitado este país puede ser comparado con él, y en muchos aspectos él es aún muy superior a ese eminente virtuoso. [...] El Senor[sic] White fue llamado dos veces [a escena], el entusiasmo trascendiendo las usuales limitaciones del aplauso para encontrar expresiones en gritos de "Bravo, bravo" cuando el interpretó una parte de una de una preciosa gavotte de $\mathrm{Bach}^{45}$.

40 Para más detalles concernientes a fechas y nombres de los solistas que actuaron con ambas orquestas, consultar Krehbiel 1892.

41 "The Thomas Symphony Concerts", The Times (Philadelphia), II/265 (14 de enero, 1876): 1.

42 La Philharmonic Society of Brooklyn fue fundada en 1857 bajo la dirección de Theodore Eisfeld (1816-1882). Desde 1861 hasta 1891 la orquesta fue dirigida por Theodore Thomas. La más reciente Brooklyn Philharmonic (activa desde 1954 a 2013) y la Philharmonic Society of Brooklyn del siglo XIX no son una continuación de la misma organización. Para más información ver Edwards 2006.

43 “Amusements. Brief mention", The New York Times, XXV/7594 (17 de enero, 1876): 4.

44 "The Philharmonic Rehearsal", The Brooklyn Daily Eagle, XXXVII/12 (15 de enero, 1876): 3.

45 "Second Philharmonic Concert", The Brooklyn Daily Eagle, XXXVII/13 (17 de enero, 1876): 3. 
El concierto también fue reseñado por el corresponsal de Dwight's en Brooklyn quien, una vez más, pareció haberse sentido encantado por el sonido de White:

El señor Joseph White, sobre su admirable interpretación al violín yo escribí no hace mucho, tocó el Concerto en E menor de Mendelssohn y de encore, una Gavotte de Bach. El gran encanto del Señor White está en la calidad del sonido que él extrae del instrumento, el cual es singularmente puro y dulce, otras cualidades sin embargo no le faltan ${ }^{46}$.

Después de haber pasado aproximadamente cuatro meses en Nueva York, el epicentro de su gira norteamericana, ciertas asociaciones personales comienzan a hacerse evidentes y explican de una forma $u$ otra el que White participara en algunos eventos específicos. El 23 de enero White actuó en la Casa-Club de la German-American Liederkranz Society de Nueva York, situada en 4th Street. Esta organización tuvo un rol importante en la promoción de la música germana en esta ciudad durante la segunda mitad del siglo XIX. William Steinway (1835-1896), uno de los dueños de Steinway \& Sons y fundador de Steinway Hall, donde White había actuado por primera vez en Nueva York -y quien asistió al primero de esos conciertos ${ }^{47}$ - era también el presidente de la Liederkranz Society ${ }^{48}$. Que White haya sido invitado a actuar para la élite cultural germanófila de la ciudad muestra el grado de estimación con que sus interpretaciones eran apreciadas. Su programa incluyó el Air Varié, op. 22, no. 2 de Vieuxtemps, su propia Styrienne, op. 11, y dos encores de Bach. Respecto de este concierto el New York Tribune tuvo solo palabras de elogio ${ }^{49}$.

Cuatro días más tarde, el 27 de enero, White participó en un concierto de la Madrigal Society de Brooklyn -en la sala del Brooklyn Athenaeum, en Atlantic Avenue y Clinton Street ${ }^{50}$-, después de esto se retiró casi un mes de los escenarios seguramente por la visita de su esposa Augustine ${ }^{51}$ quien estuvo junto a él desde el 16 de diciembre de $1875^{52}$ hasta el 19 de febrero de $1876^{53}$.

Comenzando con una actuación en la Brooklyn Academy of Music, el 23 de febrero (cuatro días después de que su esposa saliera hacia París), con el pianista S. B. Mills (1823$1898)^{54}$ y con la contralto Anna Holbrook ${ }^{55}$, White recuperó el impulso que parecía haber

46 Dwight's Journal of Music, XXXV/23 (19 de febrero, 1876): 184.

47 William Steinway's Diary (1861-1896): October 19, 1875.

48 Liederkranz New York 2018.

49 “The Liederkranz”, New-York Tribune, XXXV/10863 (24 de enero, 1876): 5.

50 The Brooklyn Daily Eagle, XXXVII/15 (19 de enero, 1876): 1.

51 Augustine White, née Augustine Rosalie Sophie Marie Adele Vivien (1848-1882). Boyadjiev 2015: 39, transcripción y traducción del certificado de matrimonio Vivien-White, del 7 de junio, 1866. Archives de Paris-V4E 1191-534.

52 "Passengers Arrived", The New York Times, XXV/7568 (17 de diciembre, 1875): 8; información adicional en Archives départementales de Seine-Maritime, Francia, y Records of the U.S. Customs Service, National Archives, EUA.

53 "Passengers Sailed". The New York Times, XXV/7323 (20 de febrero, 1876): 12; información adicional en Archives départementales de Seine-Maritime, Francia.

54 Sebastian Bach Mills (más conocido como S. B. Mills) era un pianista originario de Inglaterra, que actuaba de manera regular en Nueva York como solista con la Philharmonic Society of New York y acompañando a solistas de renombre (Bomberger 1999: 187; New York Philharmonic, Leon Levy Digital Archives; Saffle 1998: 23).

55 Este concierto fue anunciado a diario en The Brooklyn Daily Eagle, del 14 al 24 de febrero de 1876 . 
perdido temporalmente durante el período de enero-febrero y a partir de este momento sus actuaciones se volvieron más regulares y menos espaciadas entre sí.

El 12 de marzo White volvió a Steinway Hall para actuar como solista por tercera vez con The Thomas Orchestra. Este fue un concierto benéfico para ayudar a The Women's Centennial Union ${ }^{56}$. The New York Times reportó acerca de este concierto, pero descuidó mencionar los nombres de las obras interpretadas por White. Sin embargo, en pocas palabras, lo declaró un éxito ${ }^{57}$. Una segunda colaboración con la Madrigal Society de Brooklyn en el Athenaeum, el 16 de marzo, le ganó a White más halagos. El programa consistió en su propia Fantaisie sur "Traviata", su Valse de Concert, y el Rêverie, op. 22, de Vieuxtemps. El reportero de The Brooklyn Daily Eagle escribió, entusiasmado:

Del Señor White no es necesario extendernos, su interpretación, si esto fuera posible, es más exquisitamente tierna y encantadora que nunca ${ }^{58}$.

\section{EL SEGUNDO CONCIERTO CON LA PHILHARMONIC SOCIETY OF NEW YORK}

La segunda presentación de White como solista con la Philharmonic Society of New York tiene un interés histórico peculiar, al igual que lo tuvo su debut, pero por diferentes razones, esta vez relacionadas con el liderazgo de la orquesta y con la situación financiera de la institución. El concierto tuvo lugar el 18 de marzo de 1876 en la Academy of Music de Manhattan, y la dirección de la orquesta recayó sobre su concertino George Matzka (18251883). La parte del programa interpretada por White consistió en la Ballade et Polonaise de Vieuxtemps y la Ciaccona de Bach. White interpretó dos encores, primero su Styrienne y después una Gavotte de Bach. El que White interpretara a Bach durante un programa o como encore ya era algo esperado y, como bien se hace notar en el programa, la Ciaccona había sido programada "a petición" ${ }^{59}$ (ver Figura 3).

Los eventos que precipitaron la sustitución del director principal resultaron en un cambio de liderazgo de la orquesta. Ch. E. Russell, en The American Orchestra and Theodore Thomas, explica que el éxito creciente y la popularidad de la Thomas Orchestra contribuyó al deterioro de la Philharmonic Society of New York, que estaba pasando por una crisis financiera. Esa situación parece haberle provocado una gran angustia a su director titular, Carl Bergmann. También Howard Shanet, en Philharmonic, asigna el deterioro de la orquesta a una combinación de factores, incluyendo el creciente éxito de la Thomas Orchestra y la gran crisis financiera que había comenzado con el "Pánico de 1873" y que se extendió hasta 1879, afectando la economía de Estados Unidos y de varios países de Europa y Sudamérica. Durante la temporada 1875-1876 Bergmann dirigió el primero de seis programas, pero el 18 de marzo -con White como solista- él no pudo hacerlo y Matzka tuvo que reemplazarlo, lo que resultó en que la Philharmonic Society le pidiera a Bergmann su dimisión el 24 de

56 The New York Times, XXV/7631 (29 de febrero, 1876): 4. Para más información sobre The Women's Centennial Union en 1876 ver: The New York Times, XXV/7616 (11 de febrero, 1876): 5; XXV/7626 (23 de febrero, 1876): 5; XXV/7640 (10 de marzo, 1876): 8; XXV/7646 (17 de marzo, 1876): 5 .

Para más información sobre el Women's Pavilion y el National Centennial Exhibition de Filadelfia, consultar McCabe 1876.

57 New-York Tribune, XXXV/10905 (13 de marzo, 1876): 5.

58 "The Madrigal Society Concert", The Brooklyn Daily Eagle, XXXVII/65 (17 de marzo, 1876): 3.

59 Shanet (1975: 347) menciona que el éxito de White inspiró su segunda invitación con la orquesta en la misma temporada y que la Ciaccona tuvo que ser repetida "a petición". 


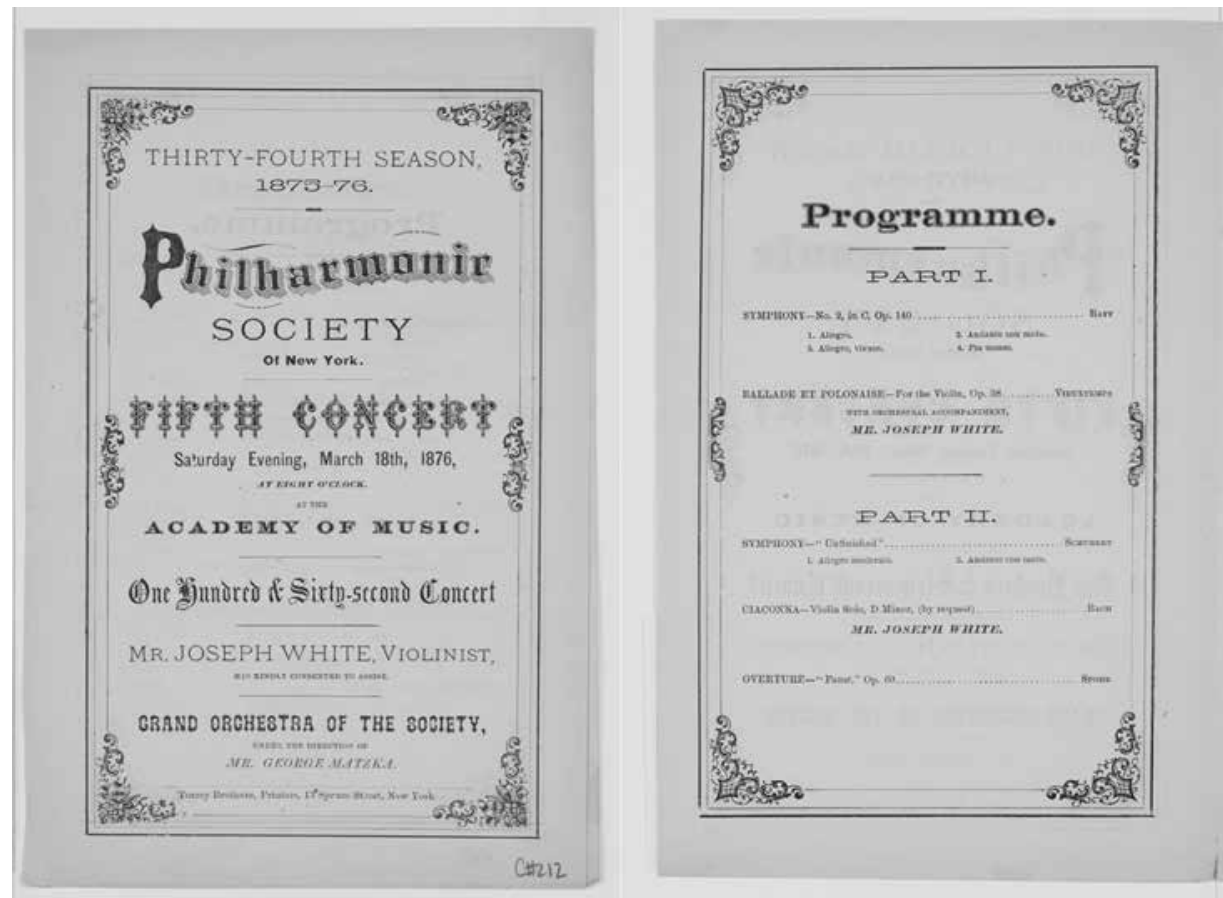

Figura 3. Programa de la segunda actuación de White con la Philharmonic Society of New York, el

18 de marzo de 1876. Academy of Music, New York. Cortesía de New York Philharmonic, Leon Levy Digital Archives, Program no. 212.

marzo. Matzka tuvo que terminar la temporada hasta el último programa del 22 de abril, con S. B. Mills de solista60.

Para la Philharmonic Society of New York, la temporada 1875-1876 fue una de las más problemáticas en cuanto a finanzas y en cuanto al personal. La mayoría de los solistas aceptaron actuar sin cobrar y la junta directiva llegó a pedirle a los músicos de la orquesta que tocaran de manera voluntaria ${ }^{61}$. White no recibió remuneración por ninguno de sus conciertos con la orquesta ${ }^{62}$. En vez de pago por su primer concierto del 11 de diciembre de 1875 , le fueron entregados una docena de tickets para el ensayo general y otra docena de tickets y asientos para el día del concierto, para sus amistades ${ }^{63}$. La disposición de White de ayudar a la orquesta por segunda vez ofreciendo otro concierto sin recibir pago alguno fue recibida con gratitud por la Philharmonic Society, la que el 24 de marzo resolvió "que

60 New-York Tribune XXXV/10911 (20 de marzo, 1876): 4; Goldberg 2013; Russell 1927: 111-112; Krehbiel 1892: 70; Shanet 1975: 153. "The Philharmonic Concerts”, The New York Times, XXV/7678 (24 de abril, 1876): 5.

61 New York Philharmonic, Leon Levy Digital Archives, Board of Directors Meeting Minutes, 18741877 (25 de marzo, 1876): 145.

62 New York Philharmonic, Leon Levy Digital Archives, Secretary's Accounts Income and Expenses Ledger 1868-1885: 64-67.

63 New York Philharmonic, Leon Levy Digital Archives, Board of Directors Meeting Minutes, 1874-1877, 3 de diciembre de 1875: 134; Business Meeting Minutes, 1866-1882 (17 de diciembre, 1875): 167-168. 
las partituras orquestales de Ballade et Polonaise con el sello de la [Philharmonic] Society en cada partitura le sean otorgadas al artista”. En esa misma reunión también se determinó pedirle a Bergmann que renunciara ${ }^{64}$.

La actuación de White provocó apasionadas reacciones positivas ${ }^{65}$ y el concierto fue reseñado en varios periódicos locales. Uno de ellos, el New-York Tribune reportó con satisfacción:

"New York Philharmonic Society". El quinto concierto de la Philharmonic Society of New York (34a temporada) se llevó a cabo el sábado por la noche. Mr. Bergmann estuvo ausente de su lugar acostumbrado y Mr. George Matzka dirigió el concierto con habilidad y compostura [...] Mr. Joseph White fue recibido con el más grande entusiasmo, el cual él justificó con su ejecución insuperable. Siempre es un placer escuchar a este refinado y simpático artista, pero nos pareció que el sábado su actuación estuvo aún más elegante y más altamente pulida que nunca. Después de una deliciosa interpretación de la Ballade et Polonaise de Vieuxtemps, él fue llamado a escena y ofreció, con acompañamiento de piano, una refinada Styrienne compuesta por él mismo, una agradable pieza exquisitamente interpretada, y después de la gran Chaconna de Bach, respondió con la misma Gavotta de Bach como encore, la cual ha tocado aquí una o dos veces. Lo bien que él conoce estas dos piezas, ya todos lo sabemos ${ }^{66}$.

Finalmente, fue ahora el Dwight's el que comparó a White y a Wieniawski, analizando los puntos fuertes y débiles de los estilos de interpretación de cada uno:

"Music in New York". [.] El solista fue Joseph White, quien ya se había presentado en uno de los conciertos de la Philharmonic más temprano en la temporada. [...]. Los méritos más sobresalientes de la ejecución del Sig. White son la precisión de sus doblecuerdas y la perfecta pureza de su entonación. Sus defectos son falta de fuerza de su arco y falta de amplitud en su estilo, lo cual a veces se sintió en su concepción de la música clásica. En él solo esas características faltan, lo cual mantiene a Wieniawski al frente de los intérpretes de esta música -la fuerza de nervios y la amplitud de su arco. Muy frecuentemente, sin embargo, tanto Wieniawski como el irlandés Ole Bull, tocan con mucha fuerza y ruido y sus pasajes rápidos usualmente raspan. El estilo de ejecución del Sig. White se caracteriza por refinamiento más que por fuerza, aunque su extraordinaria afinación hace que cada nota de su violín sea diferente y penetrante $[\ldots]^{67}$.

\section{LOS CONCIERTOS DE BOSTON}

Los siguientes conciertos llevaron a White a Boston, donde su debut tuvo lugar el 26 de marzo en el Boston Theatre, situado en Washington Street. Este debut le ganó una efusiva bienvenida en esa ciudad y gran atención por parte de la prensa local. La orquesta para la ocasión fue el grupo conocido como The Boston Theatre Orchestra o The Lothian Orchestra, dirigido por Napier Lothian (1836-1916), quien era también el director del Boston Theatre ${ }^{68}$. Varios periódicos cubrieron el evento, unos más efusivos que otros, y el

64 New York Philharmonic, Leon Levy Digital Archives, Business Meetings Minutes, 1866-1882 (24 de marzo, 1876): 172-174.

65 "New York Philharmonic Society", New York Herald, XLI/14454 (19 de marzo, 1876): 12.

66 "New York Philharmonic Society", New-York Tribune, XXXV/10911 (20 de marzo, 1876): 4

67 "Music in New York", Dwight's Journal of Music, XXXVI/2 (29 de abril, 1876): 224.

68 Pisani 2014: 127 y 339, menciona que Napier Lothian sostuvo "la más antigua dirección musical continua de un teatro [...] con el Boston Theatre, durante cuarenta años". Otras menciones de Lothian se incluyen en Trotter 1878: 178, 188; y en Franceschina 2017: 96-99, "Napier Lothian". 
consenso general fue que White había creado una impresión memorable ${ }^{69}$. The Boston Daily Globe comparó a White con el renombrado solista noruego Ole Bull (1810-1889) ${ }^{70}$, quien había actuado en Estados Unidos varias veces antes de White ${ }^{71}$.

La segunda presentación de White en la ciudad, el 2 de abril, fue igualmente aclamada ${ }^{72}$. El programa incluyó su Fantaisie sur "Martha" y el Concierto en mi menor de Mendelssohn, con la Lothian Orchestra y cantantes del Young Apollo Club ${ }^{73}$. En general, la aceptación crítica de White en Boston fue muy positiva, pero quizás la reseña más interesante es una citada por Trotter en su perfil biográfico sobre White, pues nos ofrece también una idea de su personalidad, carácter e imagen pública. Otro elemento de valor en esta reseña es que el crítico de The Musician and Artist, había seguido a White desde su comienzo en Nueva York hasta el momento de su debut en Boston y por esto puede ofrecer una valoración general de su gira. Aquí, además de la ya familiar comparación con Wieniawski, White es comparado otra vez con Ole Bull, y, además, con Henri Vieuxtemps (1820-1881):

"Música en New York". [...] Joseph White es en algunos aspectos el mejor violinista que ha visitado este país que yo recuerde, sin excepción de Wieniawski. Él y su compañero Ignasio [sic] Cervantes, pianista, hicieron su debut en esta ciudad hace algunos meses, con publicidad muy modesta, y sin anuncios sensacionalistas de los periódicos, y en el primer concierto mismo se aseguraron un éxito futuro incuestionable. Este éxito se debe enteramente a White porque, aunque Cervantes es un pianista bastante bueno, no es nada maravilloso. Pero White fue una revelación. Su primera actuación frente a la gran audiencia general de New York fue durante un concierto con la Philharmonic (cuya fecha ahora no puedo recordar), cuando tocó el concierto de Mendelssohn y la chacona de Bach. El concierto de Mendelssohn fue excelente, especialmente el último movimiento; pero fue en la chacona de Bach la que mostró cuán realmente bueno él era. Yo he escuchado esta composición de todos los violinistas eminentes (excepto Vieuxtemps) que han visitado nuestra ciudad, pero nunca escuché una interpretación tan satisfactoria. Las tres voces fluyeron de manera tan suave y uniforme, que nunca parecían interponerse entre sí: siempre parecía existir suficiente cantidad de arco y siempre en el lugar correcto para que cada voz individual recibiera exactamente su debida importancia. La vociferante reacción que le siguió a esta valiosa interpretación fue bien merecida. White es un mulato cubano, de aspecto elegante y extremadamente caballeroso en apariencia y en conversación. Un escritor de Brooklyn habla de él de la siguiente manera: "Su estilo es la perfección en sí misma; su arco es magnífico y su tono exquisito. Su ejecución es mejor que la de Ole Bull; posee más sentimiento que Wieniawski; y el volumen de su sonido es mayor que el de Vieuxtemps “, con todo lo cual yo estoy de acuerdo ${ }^{74}$.

69 "Local Summary", Boston Post, XC/74 (27 de marzo, 1876): 5; "Sunday Concert at the Boston Theatre", Boston Post, XC/78 (31 de marzo, 1876): 3.

70 Bergsagel 2001.

71 "MUSICAL. Sunday Evening Concerts", The Boston Daily Globe, IX/74 (27 de marzo, 1876): 3, citado en Trotter 1878: 237.

72 "At the Boston Theatre", The Boston Daily Globe, IX/80 (3 de abril, 1876): 4.

73 "The Sunday Concert at the Boston Theatre", Boston Post, XC/79 (1 de abril, 1876): 3.

74 "Music in New York", The Musician and Artist (Boston), I/3 (marzo, 1876): 84-88, citado en Trotter 1878: 236. Para más reseñas acerca del debut de White en Boston el 26 de marzo de 1876, ver Trotter 1878: 236-240, que incluye además reseñas en [Boston] Daily Globe, Boston Journal, Herald, Daily Advertisery The Daily Evening Transcript, e indica que todas son del 27 de marzo de 1876. 


\section{DE VUELTA A NUEVA YORK Y A BROOKLYN75}

Después de sus dos presentaciones en Boston, White regresó a Nueva York y a sus alrededores, actuando otra vez con el pianista S. B. Mills, el violista Julius Risch, y el chelista Charles Warner en Staten Island, en la Association Hall, situada en West New Brighton ${ }^{76}$. Durante el resto de abril y mayo de 1876 White actuó mayormente en Brooklyn, también en Manhattan, y solo una vez en Boston. Durante este período él colaboró mayormente con cantantes y con su compatriota Emilio Agramonte, el que actuó como su pianista acompañante.

La mayoría de los conciertos de esta parte de su gira fueron de carácter benéfico, para ayudar a iglesias, a niños, a dos amigos cantantes y a dos hospitales. Dos de estos conciertos fueron el 12 de abril y el 22 de mayo en Plymouth Church, en Brooklyn. El propósito del primer concierto fue ayudar a recaudar dinero para el Betel de la iglesia ${ }^{77}$. Aunque la reseña aparecida en The Brooklyn Daily Eagle no menciona a ningún otro músico que haya colaborado con White, es muy posible que haya actuado junto a Emilio Agramonte, ya que él repitió este mismo programa con dicho músico ocho días después también en Brooklyn ${ }^{78}$.

El segundo concierto en Plymouth Church fue para recaudar fondos para la cantante Clementine Lasar (s/d-1929), soprano que pertenecía a esa iglesia ${ }^{79}$. La asociación de White con esta congregación religiosa -fundada en 1847- es de interés especial, pues Plymouth Church no era solo una de las iglesias de más recursos económicos en Estados Unidos, sino también un centro antiesclavista de reconocida influencia durante la Guerra Civil, aunque continuó siéndolo durante el resto del siglo XIX y posteriormente se convirtió en un centro de activismo y apoyo a los derechos de las personas de ascendencia africana. El primer pastor de la iglesia fue Henry Ward Beecher (1813-1887), uno de los más prominentes y activos defensores de la abolición de la esclavitud en Estados Unidos ${ }^{80}$. Debido a la afinidad de White por la causa abolicionista, es muy posible que él haya asistido a alguno de los sermones de Beecher y también que hiciera amistad con varios miembros de la congregación como Sigismond Lasar (1822-1896), organista de renombre en Plymouth, profesor de música, compositor y prominente abolicionista ${ }^{81}$.

Las colaboraciones con Emilio Agramonte llevaron a White, "el celebrado violinista", a la Brooklyn Academy of Music el 20 de abril, como parte de un evento con la Handel and Haydn Society ${ }^{82}$, y a Chickering Hall en Manhattan, el 4 de mayo, como parte del concierto que Agramonte organizaba anualmente para sus alumnos de canto (ver Figura 4). En este evento Agramonte estrenó partes de su propio Stabat Mater e interpretó con White la Fantasia Appassionata, op. 35, de Vieuxtemps ${ }^{83}$.

75 Para esta sección de la gira no he agrupado los eventos cronológicamente. Para una lista cronológica ver el Calendario de Conciertos al final de este artículo.

76 Staten Island NY Evening Post, (abril, 1876), (s/f, s/p), en www.fultonhistory.com, [último acceso noviembre de 2017].

77 The Brooklyn Daily Eagle, XXXVII/81 (5 de abril, 1876): 1.

78 "Tableaux and Music", The Brooklyn Daily Eagle, XXXVII/88 (13 de abril, 1876): 4.

79 “Miss Lasar's Concert”, The Brooklyn Daily Eagle, XXXVII/120 (20 de mayo, 1876): 4.

80 Existen numerosas referencias pertinentes al reverendo Beecher en la prensa de este período.

81 "Obituary record Prof. Sigismond Lasar", The New York Times, XLIV/13749 (15 de septiembre, 1895): 5 .

82 "Handel and Haydn Society. Grand Concert", The Brooklyn Daily Eagle, XXXVII/93 (19 de abril, 1876): 1. Al momento de la gira de White existían varias organizaciones musicales locales con el nombre "Handel and Haydn Society". La Handel and Haydn Society de Brooklyn no debe confundirse con la Handel and Haydn Society con sede en Boston que todavía permanece activa desde su fundación en 1815 .

83 Brooklyn Historical Society, Clementine Lasar Studwell Scrapbook (programas). 
Durante este período de la gira White también realizó un corto viaje a Boston para tomar parte, el 8 de mayo, en un concierto benéfico para ayudar a una cantante que iba a viajar a Europa ${ }^{84}$. También actuó dos veces más en Chickering Hall por razones benéficas, primero para ayudar a una iglesia rural, el 17 de mayo ${ }^{85}$, y segundo, el 12 de junio, para ayudar a construir un hospital que estaría dedicado a la cura de enfermedades crónicas ${ }^{86}$. Otras actuaciones en iglesias de Brooklyn incluyeron la Cuyler Presbyterian Church el 25 de abril ${ }^{87}$, la Hanson Place Baptist Church el 16 de mayo ${ }^{88}$ y un concierto en Saint Ann's Church el 21 de mayo, para contribuir a una asociación de ayuda a niños y niñas. En este último interpretó, junto con sus asiduos colegas Mills, Werner y Dachauer, un Offertory de Marie Grandval (1828-1907) ${ }^{89}$. Antes de despedirse para siempre de Brooklyn, White tomó parte en dos conciertos más: el primero en Bedford Avenue Reformed Church el 29 de mayo $^{90}$ y el segundo en el Brooklyn Athenaeum, el 1 de junio ${ }^{91}$.

\section{LOS ÚLTIMOS TRES CONCIERTOS: FILADELFIA}

Los conciertos finales de la gira de White en Estados Unidos fueron en junio de 1876 en la ciudad de Filadelfia y tuvieron lugar en la Academy of Music en South Broad Street. Filadelfia estaba en ese momento en plena actividad con motivo de la primera feria internacional celebrada en el país, en este caso para celebrar los cien años de la Declaración de Independencia de Estados Unidos. Las celebraciones de la feria, también conocida como "Centennial Exhibition", duraron desde mayo hasta noviembre de 1876 y trajeron consigo una multitud de invitados no solo de todas partes de Estados Unidos sino también representaciones oficiales de países de todo el mundo ${ }^{92}$. Uno de los dignatarios internacionales que formaron parte de las comisiones extranjeras acreditadas en los eventos fue el emperador de Brasil Dom Pedro II (1825-1891) ${ }^{93}$ quien, junto con su esposa la emperatriz Teresa Cristina (1822-1889) y al entonces presidente de Estados Unidos Ulysses Simpson Grant (1822-1885), participó en la ceremonia inaugural del 10 de mayo ${ }^{94}$.

84 "Boston Music Hall”; "Music Notes", The Boston Daily Globe, IX/108 (6 de mayo, 1876): 4.

85 "[...] for the benefit of a country church", New York Herald, XLI /14512 (16 de mayo, 1876): 9.

86 "Music for Chronic Diseases", New York Herald, XLI/14528 (1 de junio, 1876): 5.

87 The Brooklyn Daily Eagle, XXXVII/96 (22 de abril, 1876): 1. La iglesia era también conocido como "Dr. Cuyler's Church" en referencia al ministro Theodore Ledyard Cuyler (1822-1909).

88 The Brooklyn Daily Eagle, XXXVII/116 (16 de mayo, 1876): 1.

89 "Sacred Concert at St. Ann's Church", New-York Tribune XXXVI/10965 (22 de mayo, 1876): 4.

90 "Open Air Fund Concert", The Brooklyn Daily Eagle, XXXVII/128 (30 de mayo, 1876): 3.

91 "Grand Thursby Concert", The Brooklyn Daily Eagle, XXXVII/126 (27 de mayo, 1876): 1; "Thursby Concert To-night" y "Madrigal Society Charity Concert”, The Brooklyn Daily Eagle, XXXVII/127 (29 de mayo, 1876): 4 .

92 Para más información acerca de las celebraciones del Centennial ver McCabe: 1876 y The Library Company 2008.

93 Dom Pedro II y su familia se convirtieron con el tiempo en personas muy importantes para White. Este vivió en Brasil desde 1879 hasta 1889. Durante esos años, fue el maestro de música de la hija del emperador, la princesa Isabel, y de sus hijos. El emperador lo condecoró con honores reales. La amistad entre White y la familia imperial se extendió hasta el final de sus vidas.

94 Thompson 2010. Para más información de las actividades de Dom Pedro II durante este período ver The New York Times XXV/7665 (9 de abril, 1876): 12; "Dom Pedro's Arrival", The New York Times XXV/7671 (16 de abril, 1876): 1; "The Emperor of Brazil", The New York Times XXV/7691 (9 de mayo, 1876): 1. 
El momento y la sede de la serie musical "The Musical Congress" no pudieron haber sido más propicios. Su organizador, el empresario James W. Morrissey ${ }^{95}$, tomó en cuenta todo lo que la ciudad podía ofrecerle en ese momento -el Centennial, la presencia de Dom Pedro II, el gran influjo de delegaciones internacionales de alto perfil y el público cosmopolita- para promocionar su concierto operático de "estrellas". Los tres conciertos se vendieron completamente y, en un último truco publicitario, él promovió -el día del último concierto- que mil sillas adicionales fueran agregadas en el escenario, las que resultaron vendidas ese mismo día ${ }^{96}$.

Los solistas contratados por Morrissey eran miembros de varias compañías de ópera como la Grand Italian Opera Company, administrada por Max Strakosch (1835-1892), y la muy popular English Opera Company, administrada y liderada por Clara Louise Kellogg (1842-1916) ${ }^{97}$. Estos solistas fueron Pasquale Brignoli (1842-1884), Zelda Seguin (18481914), Franz Remmertz (1845-¿̇), Annie Louise Cary (1842-1921), además de otros músicos extranjeros y estadounidenses de renombre, como la pianista norteamericana Julie Rivé King (1854-1937), el ya mencionado pianista S. B. Mills -quien era el maestro de Rivé-, la arpista de origen catalán Esmeralda Cervantes [née Clotilde Cerdá] (1852-1926), arpista de la Casa Imperial de Río de Janeiro que estaba en la ciudad junto con la delegación de la familia imperial de Brasil ${ }^{98}$ (a cuya petición fue integrada al elenco) ${ }^{99}-\mathrm{y}$, por supuesto, José White. El evento también incluyó al bajo Signor Ferranti, a Eugenie Palmiri y a los directores musicales Max Maretzek (1821-1897) ${ }^{100}$, Louis Dachauer, E. Berhens y George W. Colby, además de una orquesta no mencionada en los anuncios de prensa, y un coro agregado el último día de la serie ${ }^{101}$.

White y Mills abrieron el concierto inicial de la serie el 19 de junio, con la Sonata "Kreutzer", op. 47, de Beethoven. Es difícil concluir si White y Mills interpretaron la obra completa o decidieron omitir el Presto del primer movimiento para acortarla -la sonata completa dura aproximadamente cuarenta y cinco minutos-, ya que los tres movimientos no están correctamente identificados. La segunda contribución de White fue el Ave María de Gounod con Esmeralda Cervantes en el arpa -un seguro éxito de audiencia-, y por último, una pieza para solista de Vieuxtemps identificada en el programa como "Air et Varié", posiblemente un error de impresión en vez del más preciso título Air Varié, en su versión orquestal, aunque la orquesta no se menciona en el programa (ver Figura 4) ${ }^{102}$ :

95 Hines and Hanaford 1914: 231, contiene una entrada biográfica de "James W. Morrissey" (sin fechas de nacimiento y muerte).

96 The Times (Philadelphia) II/404 (24 de junio, 1876): 3.

97 Preston 2017: 184-185.

98 Biblioteca de Catalunya 2012, Guzmán 1876.

99 Morrissey 1904:109.

100 Franceschina 2017: 142-144.

101 Dwight's Journal of Music, XXXVI/918 (24 de junio 1876): 255.

102 En el catálogo de composiciones de Henri Vieuxtemps no existe pieza alguna con el nombre "Air et Varié". Existen sin embargo dos Air Varié: (1) Air Varié avec Introduction d' opéra "Il Pirata" de Bellini en re mayor, op. 6, para violín y piano u orquesta (ca. 1837); y (2) la segunda pieza del ciclo Six Morceaux de Salon, op. 22, Air Varié en re menor, también escrita para violín y piano u orquesta ( $c a$. 1846-1847). Radoux 1891: 189-190; Petrucci Music Library 2016. Aunque los anuncios del concierto no mencionan orquesta alguna, sabemos por Morrissey (1904: 104) que existió una orquesta que acompañó a los solistas. 


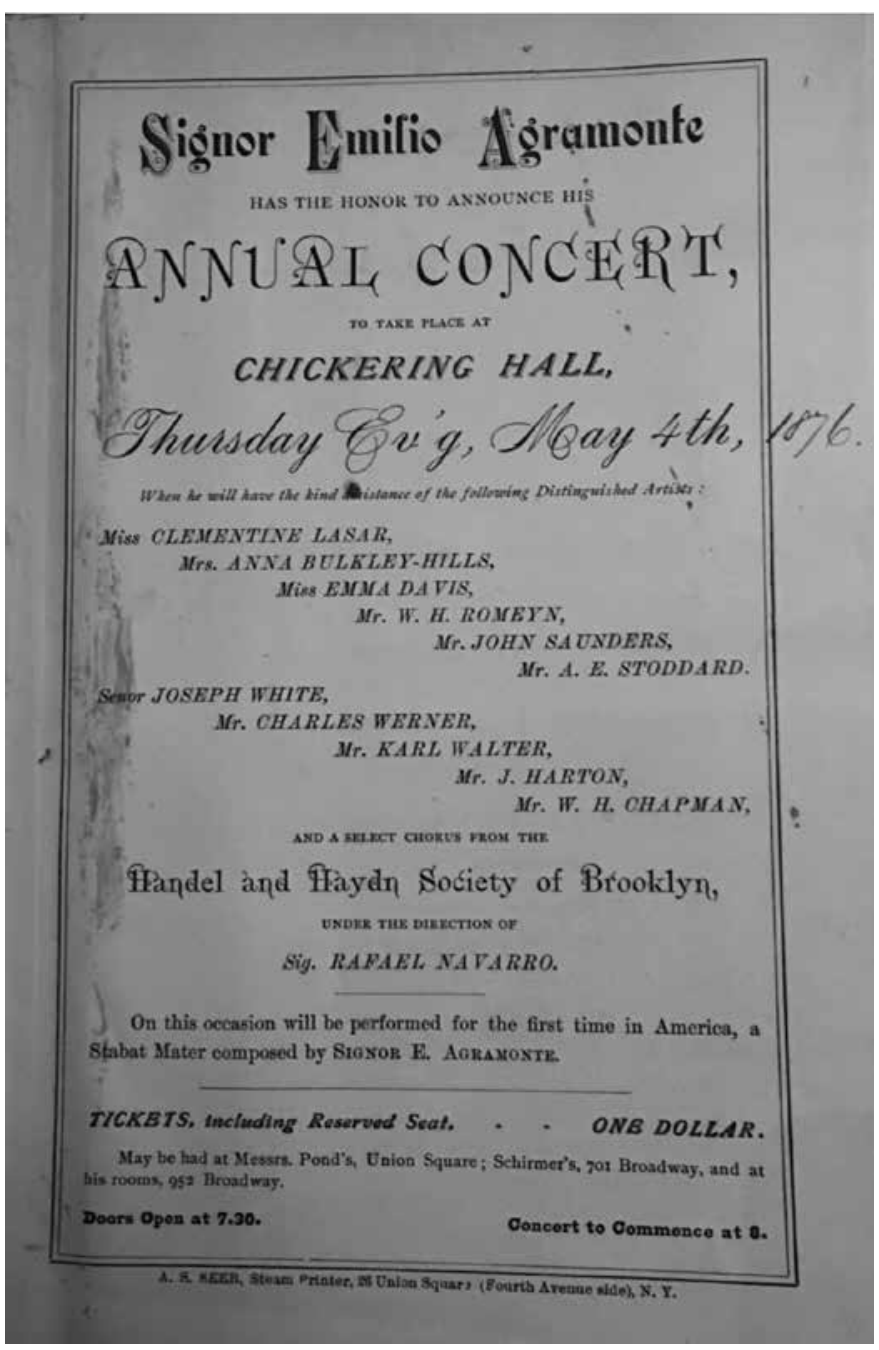

Figura 4. Programa del concierto "Signor Emilio Agramonte Annual Concert", el 4 de mayo de 1876. Chickering Hall, New York. Cortesía de Brooklyn Historical Society.

Clementine Lasar-Studwell Scrapbook: Programs.

En el segundo concierto, el 21 de junio, White interpretó la Grande fantaisie de concert sur "Robert le Diable", op. 44, de Alard. Esta obra, para violín y piano o violín y orquesta, al parecer fue interpretada aquí en su versión orquestal, ya que no se anunció pianista alguno (ver Figura 5). El New York Clipper publicó una reseña positiva de los dos primeros conciertos ${ }^{103}$.

103 "Musical", New York Clipper, XXIV/14 (1 de julio, 1876): 6. 


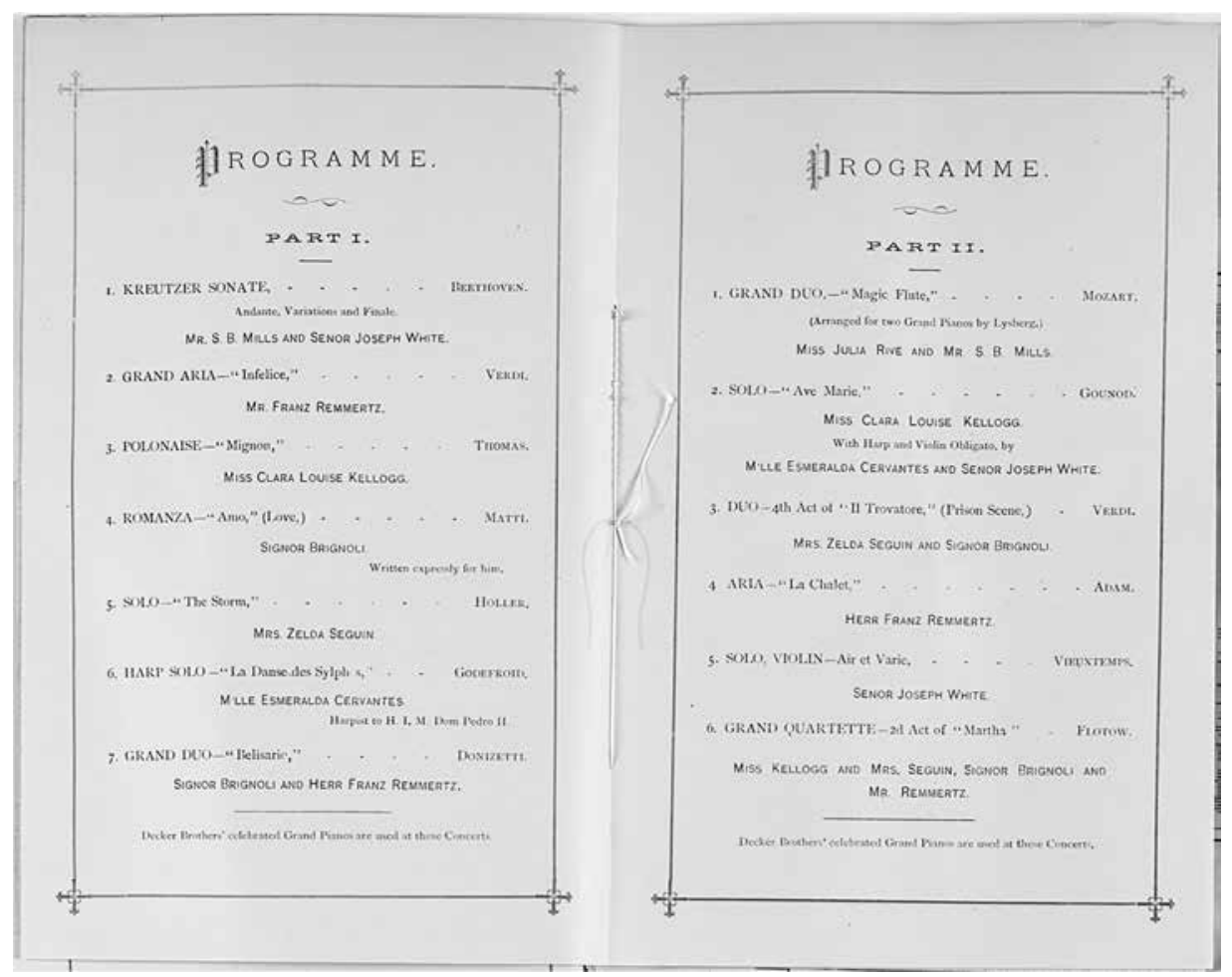

Figura 5. Primer programa de la serie "The Musical Congress", del 19 de junio de 1876. Academy of Music, Philadelphia. Historical Society of Pennsylvania, Collection 3150, Box 127, Scrapbook (1876-1879).

El último concierto de la serie coincidía con el fin de la temporada de conciertos y Morrissey lo promovió como la última oportunidad posible de escuchar a los distinguidos solistas, muchos de estos viajarían próximamente a Europa. Lo tituló "Farewell”, nombró como patrocinador del concierto a la Casa Real de Brasil y especificó que el "Congreso" le diría adiós a la audiencia norteamericana ${ }^{104}$. Además de la ya habitual presencia del emperador Dom Pedro II y de su esposa, a este último concierto asistieron miembros de las delegaciones de España y Perú que estaban en la ciudad por las actividades del Centennial. En esa gala, que fue también el último concierto de White en Estados Unidos, él interpretó otra vez el "Air et Varié", Air Varié de Vieuxtemps. Además, interpretó con Esmeralda Cervantes el Grand Duo para violín y arpa de Labarre y Beriot (ver Figura 6).

Una vez concluido el "Musical Congress", Dwight's publicó una reseña general de la serie. Era casi de esperar que este tipo de evento tan pomposo no hubiera sido del agrado del crítico, quien pudo haber sido el mismo John Sullivan Dwight (1813-1893). Este, evidentemente disgustado, escribió sarcásticos comentarios dirigidos a casi todos los solistas, también criticando a White, aunque no le destinó a este último las críticas más ásperas:

104 "Academy of Music", The Times (Philadelphia), II/403 (23 de junio, 1876): 3; "The Musical Congress", The Times (Philadelphia), II/404 (24 de junio, 1876), p. 1; "Under Imperial Patronage", The Times (Philadelphia), II/404 (24 de junio, 1876): 3. 


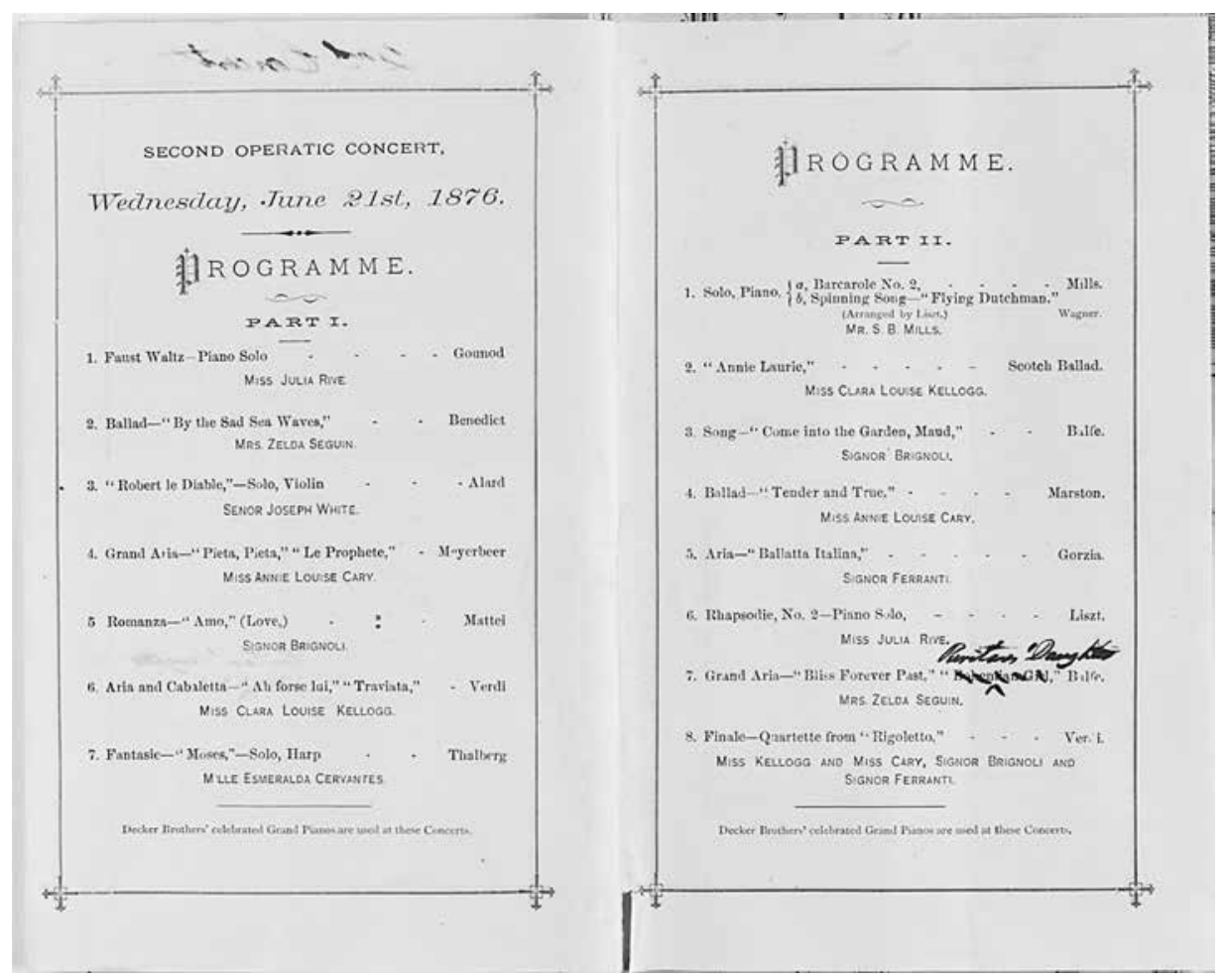

Figura 6. Segundo programa de la serie "The Musical Congress", del 21 de junio de 1876. Academy of Music, Philadelphia. Historical Society of Philadelphia, Collection 3150, Box 127, Scrapbook (1876-1879).

El Congreso Musical. Los grandes conciertos operáticos presentados en la Academia de Música por Mr. James W. Morrisey, como yo predije en mi última carta, tuvieron un gran éxito [...] Mlle. Cervantes es una agradable señorita de dieciséis años, arpista de H.I.H Dom Pedro; se viste magníficamente, luce un número de medallas; pero en honor a la verdad, yo no puedo decir mucho sobre ella en alabanza como artista; quizás a su debido tiempo ella llegará a ser una intérprete, al presente su ejecución no es más que mediocre. Mr. Joseph White, el violinista cubano, fue un hit con la audiencia, su sonido es puro y dulce pero no potente, tiene una bella técnica y ejecución considerable, pero le falta amplitud de concepción y profundidad de sentimientos. El Signor Brignoli ha visto mejores días, pero no lo sabe $[\ldots]^{105}$.

El emperador Dom Pedro II, patrón de las artes y de pensamiento abolicionista, resultó posteriormente ser una figura crucial en la vida y carrera de White, por eso es interesante indagar acerca de cómo pudieron haberse conocido. Es muy posible que el emperador haya sabido de la existencia de White antes de conocerlo y también que White conociera las ideas proarte y abolicionistas del monarca no solo por la prensa sino también por medio 
de L. M. Gottschalk, quien vivió en Brasil y era bien conocido de la familia imperial ${ }^{106}$. También existe la posibilidad de que el emperador y White se hayan conocido por intermedio de miembros de la casa de Braganza (a la que pertenecía la familia imperial) que residían en París. Según las palabras de Morrissey, el emperador ya conocía de nombre a todos los solistas del grupo ${ }^{107}$. En cuanto a las circunstancias en que se llevó a cabo el primer encuentro, si no se habían conocido antes, este pudo haber ocurrido el 19 de junio de 1876, en una recepción después del primer concierto de la serie ${ }^{108}$. Es muy posible que durante su estancia en Filadelfia y antes de marcharse de Estados Unidos, White ya hubiera recibido una invitación formal por parte de la familia imperial para viajar a Brasil con posibles planes de trabajo. También es probable que durante su estadía en Filadelfia White tuviera la oportunidad de conocer a otras personalidades representantes de gobiernos de algunos países de América Latina, a los que visitaría durante su gira de 1877-1879 (ver nota al pie 8).

Después de terminar la temporada de conciertos 1875-1876, al igual que su gira, White salió hacia París el 8 de julio de 1876, a bordo del vapor Canada vía a Le Havre, arribando a su destino once días después, el 19 de julio ${ }^{109}$. A pesar de que el New-York Tribune reportó que White volvería en septiembre, él nunca volvió a Estados Unidos ${ }^{110}$.

\section{REPERTORIO Y ESTILO DE INTERPRETACIÓN}

White era un músico de su época y su repertorio incluía las obras que se esperaban en el repertorio de los virtuosos europeos. Durante esta gira, sus propias fantasías operáticas fueron un hit tanto con la audiencia como con los críticos durante sus primeras presentaciones. Estas obras eran efectivas, ya que garantizaban el éxito con el público con poco tiempo de ensayos. Sin embargo, su repertorio no solo incorporaba obras de la tradición franco-belga del violín, sino también de la tradición germánica. Not a showman ${ }^{111}$, White no solo interpretaba sus propias obras, sino también, para lograr mayor balance en el repertorio, a compositores canónicos como Bach, Beethoven, Mendelssohn, Schumann y otros que no eran virtuosos itinerantes.

White no interpretó su propio Concierto para violín y orquesta en fa\# menor, op. 15 (1864), durante su gira norteamericana. Es posible que él tuviera que adaptarse a la programación conservadora de la Philharmonic Society of New York, de The Thomas Orchestra, y de las otras orquestas con las que actuó, las que no se arriesgaban mucho en cuestiones de repertorio con compositores desconocidos y mantenían los programas más o menos dentro de la tradición germánica (esto no debe sorprender, ya que Bergmann y Thomas eran de origen alemán) ${ }^{112}$. Sin embargo, para White no significó un problema atenerse

106 Gottschalk 2006: 320, 400-407. Gottschalk murió en Brasil en 1869.

107 Morrissey 1904: 106.

108 Morrissey 1904: 107. D’Arcier 1997: 145, sugiere que White conoció a Dom Pedro II el 4 de julio en Filadelfia, por medio de un amigo uruguayo, del que no menciona nombre. La autora no ofrece fuente alguna ni argumento que valide esta aserción.

109 "Passengers sailed", The New York Times, XXV/7743 (9 de julio, 1876): 12; información adicional en Archives départementales de Seine-Maritime, Francia.

110 New-York Tribune, XXXVI/11006 (8 de julio, 1876): 4.

111 Trotter 1878: 240.

112 Una mirada a los programas de Thomas y Bergmann de varias temporadas revela que ambos conductores favorecían a los compositores germánicos, en un segundo orden de preferencia ellos programaban a compositores franceses, y después a todos los demás incluyendo ocasionalmente a 
a dicha programación, pues él estaba interesado en establecerse como solista y para esto nada mejor que interpretar los concerti y piezas de concierto ya populares entre la audiencia, junto con instituciones musicales que contaban con un público regular. La opción de autofinanciar la puesta en escena de su composición podría haberle resultado a la vez demasiado costosa y riesgosa.

Lo que sigue es una lista del repertorio que White incluyó durante su gira de 1875-1876 en Estados Unidos. Esta lista, que no pretende ser exhaustiva, contiene la información extraída de las referencias de prensa y de los programas que he podido localizar:

Alard: Grande Fantaisie de Concert sur "Robert le diable"

Bach: Ciaccona (Partita II en re menor, para violín solo)

Bach: Gavotte en Rondeau (Partita III en mi mayor, para violín solo)

Beethoven: Sonata para piano y violín no. 9, op. 47, "Kreutzer"

Beethoven: Sonata para piano y violín no. 3, op. 30

De Grandval, Marie Clémence: Offertory

Gounod: Ave Maria

Labarre y Beriot: Grand Duo "La Muta di Portice"

Mendelssohn: Concerto para violín en mi menor, op. 64

Mendelssohn: Cuarteto para piano y cuerdas en si menor, op. 3

Mendelssohn: Piano Trio en do menor, no. 2, op. 66

Raff: Cavatina

Vieuxtemps: Air Varié (op. 6 u op. 22, no. 2)

Vieuxtemps: Ballade et Polonaise, op. 38

Vieuxtemps: Fantaisie Concertante sur "Les Huguenots"

Vieuxtemps: Fantasia Appassionata, op. 35

Vieuxtemps: Rêverie (6 Morceaux de salon, no. 3, op. 22)

White, Fantaisie sur "La Traviata", op. 4.

White, Fantaisie sur "Martha", op. 24.

White, Introduction et Variations sur le Carnaval de Venise

White, Styrienne, op.11.

White, Valse de Concert

\section{CONCLUSIONES}

La gira estadounidense de 1875 a 1876 fue importante para expandir la reputación de White fuera de Francia y también para elevar su estatura profesional y colocarlo dentro de un grupo de violinistas renombrados como Wieniawski - con quien fue repetidamente comparado-, Vieuxtemps y, su compañero de estudios, Sarasate, quienes habían actuado en Estados Unidos antes que él.

Durante el tiempo que estuvo en Estados Unidos pudo establecer amistad con la comunidad de cubanos expatriados que vivía en Nueva York, lo que le permitió reconectarse y unir fuerzas con sus coterráneos para prestar su talento y ayudar al movimiento independentista y abolicionista en Cuba. Sus colaboraciones y asociaciones con iglesias y con 
personas que tenían un rol activo en promover el mejoramiento de la vida de las personas de ascendencia africana durante y después de la Guerra Civil, demuestra que su línea de pensamiento no estaba limitada a la abolición de la esclavitud en Cuba, sino que se extendía a todos los países del mundo donde existiera la desigualdad racial.

Socialmente, White fue aceptado por el mundo musical a pesar de ser descendiente de africanos, y este es un hecho significativo, porque la sociedad en Estados Unidos estaba altamente segregada a solo diez años de la Guerra Civil. Sin embargo, es importante recordar que además de su talento y nivel como solista, su condición de extranjero automáticamente lo situaba en una categoría privilegiada en la sociedad norteamericana de entonces, lo que atenuó las consecuencias que hubiera podido sufrir por su ascendencia africana en comparación con los afroamericanos.

La presencia de personas de ascendencia africana en la escena de la música de conciertos era extremadamente minoritaria y esto hizo que su debut con la Philharmonic Society of New York, ahora New York Philharmonic Orchestra, fuera de significancia histórica -aunque esto sea significativo retrospectivamente, pues en su momento no parece habérsele definido como tal-. De hecho, es posible que White fuera el primer solista de ascendencia africana con casi todas las orquestas con las que actuó, incluyendo The Thomas Orchestra durante esta gira.

White actuó en las salas más importantes de las ciudades que visitó (Steinway Hall, Chickering Hall, las Academy of Music de Brooklyn, Manhattan, y Filadelfia, el Boston Theatre, etc.), las mismas salas donde actuaban solistas de la talla de Ole Bull, Sivori, Wieniawski, Anton Rubinstein, Teresa Carreño y muchos otros. Sus colaboraciones musicales dan una idea de la calidad de los músicos con quienes él se asoció, en qué círculos musicales se desenvolvía y cuáles eran sus conexiones personales. La mayoría de los músicos con quienes colaboró eran solistas de visita o directores de orquesta que habían emigrado de Europa y se habían radicado en Estados Unidos.

Los anuncios iniciales de la prensa a su llegada a Nueva York, para presentarlo por primera vez al público que no lo conocía, sugiere que la información obtenida por la prensa provenía del mismo White o de algún agente que lo estuviera representando en ese momento. Aunque era común que los solistas y sus empresarios recurrieran a exageraciones y a exclamaciones grandilocuentes para vender entradas, los anuncios de conciertos y reseñas -sumado a la mención específica en The Musician and Artist donde se describe su estilo de promoción como "muy modestamente promocionado" y "[sin] lenguaje sensacionalista en los periódicos" (ver nota al pie 74)-, sugieren que White no se adhirió a esta práctica.

La gira de White por Estados Unidos fue significativa para consolidar su carrera como solista. A pesar de su éxito profesional, no hay suficiente evidencia para valorar si fue lucrativa o no. Los únicos registros financieros que pude localizar, los libros de registros de la Philharmonic Society of New York, indican que White actuó dos veces sin remuneración alguna. Es muy posible que, para impulsar su carrera y al no ser un artista completamente establecido como era el caso de Wieniawski, con quien con frecuencia fue comparado, él no priorizara la ganancia económica, y que para esto hubiera autofinanciado su gira sin que el dinero hubiera sido, en general, su primera prioridad. Trotter parece respaldar esta idea cuando dice que White no estaba enfocado en hacer dinero ${ }^{113}$. No sabemos si la gran cantidad de conciertos benéficos para los que prestó su talento eran remunerados o no, sin embargo, es posible que obtuviera alguna remuneración por ellos. En el mejor de los casos, es posible que después de haber pagado todos sus gastos de estancia en Estados Unidos White haya logrado salir de este país sin ganancias ni pérdidas. 
Su aporte a causas dignas que le resultaban afines (hospitales, mujeres y niños, programas comunales, e individuos) durante esta gira, contribuyeron de alguna manera al mejoramiento de la sociedad norteamericana. El gran número de conciertos benéficos en los que participó sugiere un carácter generoso y un genuino deseo de ayudar a otros.

En cuanto a su producción musical, yo no pude encontrar ninguna indicación de que White hubiera compuesto o publicado ninguna obra nueva durante este período. Es posible que todos sus esfuerzos durante esta gira hayan estado enfocados en sus actividades como solista. Sin embargo, esto no descarta la posibilidad de que haya escrito alguna pieza en estos años.

Aunque a lo largo de su vida White compuso piezas que incorporaban el carácter nacional de los países que él visitaba, no existe indicación alguna de que él haya compuesto música inspirada en temas nacionales de Estados Unidos.

Finalmente, esta gira pudo haber sido la ocasión en la que White conoció al emperador Dom Pedro II y que este hecho lo haya impulsado hacia la siguiente gran etapa de su carrera: su gira latinoamericana y su importante estancia de diez años en Brasil.

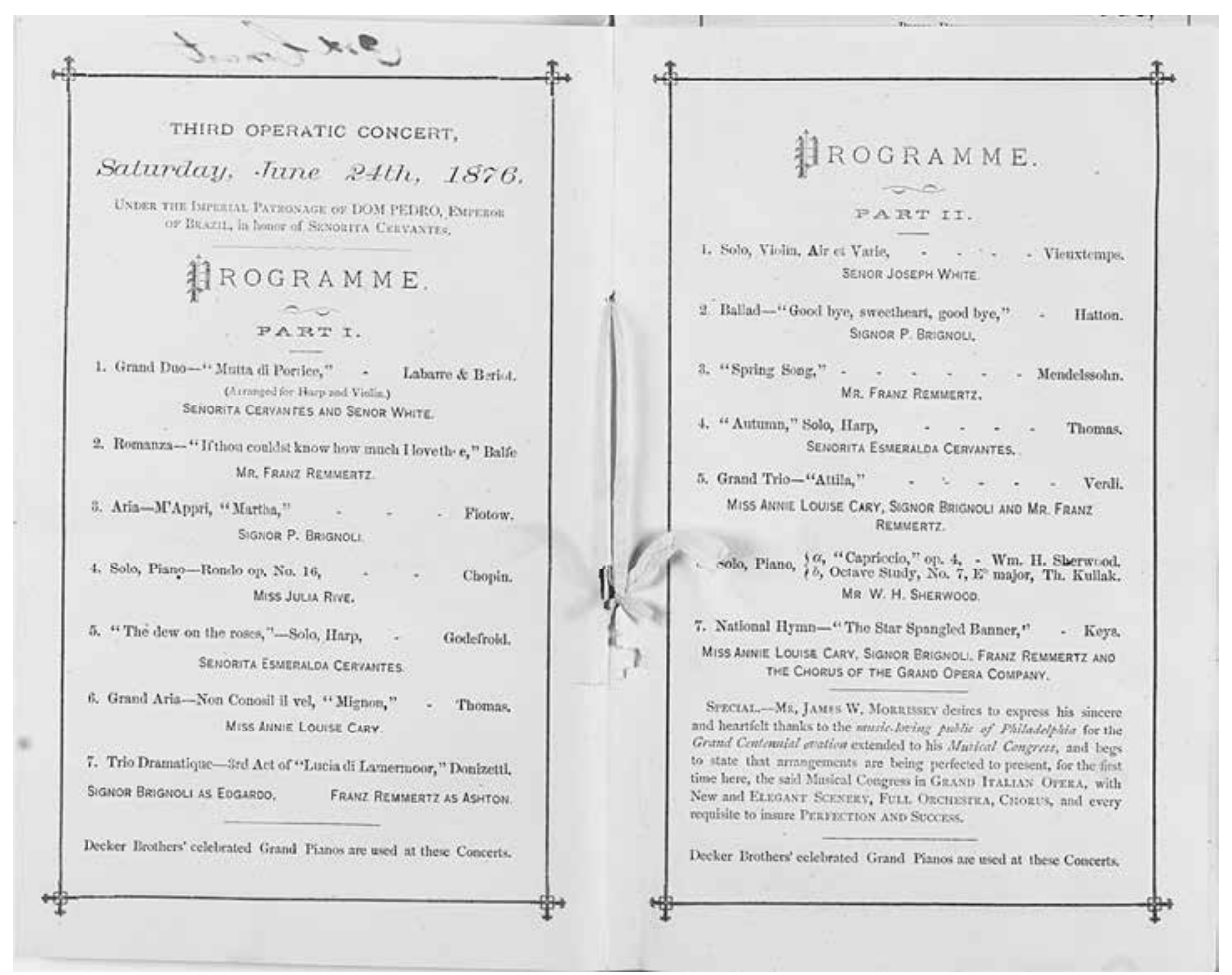

Figura 7. Programa final de la serie "The Musical Congress", del 24 de junio de 1876,

"The Last Grand Musical Concert". Academy of Music, Philadelphia. Historical Society of Philadelphia, Collection 3150, Box 127, Scrapbook (1876-1879). 


\section{BIBLIOGRAFÍA}

Bergsagel, John

2001 "Bull, Ole", Grove Music Online. www.oxfordmusiconline.com/grovemusic/view/10.1093/ gmo/9781561592630.001.0001/omo-9781561592630-e-0000004295 [acceso: 23 de junio, 2018].

Biblioteca de Catalunya

2012 "Esmeralda Cervantes: la restauració del seu Àlbum", Tríptic exposició Esmeralda Cervantes, Aitor Quiney (curador) [versión PDF], Biblioteca de Catalunya, www.bnc.cat/esl/Visitanos/ Exposiciones/Esmeralda-Cervantes-la-restauracio-del-seu-Album [acceso: 9 de enero, 2018].

Bomberger, E. Douglas (EDitor)

1999 Brainard's Biographies of American Musicians. Westport, CT: Greenwood.

BoyadjIEv, Yavet

2015 "José White Laffita (1835-1918): A Biography and a Study of his Six Études, op. 13". DMA Diss., The City University of New York.

2018 "Joseph White: Breaking New Ground", The Strad, 129/1538 (junio), pp. 44-48.

Brooklyn Historical Society

2018 "Guide to the Brooklyn Bureau of Community Service records ARC.129", Brooklyn Historical Society, http://dlib.nyu.edu/findingaids/html/bhs/arc_129_brooklyn bureau_community_service/ [acceso: 20 de junio, 2018].

D'Arcier, SAbine Faivre

1997 José White y su Tiempo. La Habana: Editorial Letras Cubanas.

EDWARds, Maurice

2006 How Music Grew in Brooklyn: A Biography of the Brooklyn Philharmonic Orchestra. Lanham, MD: Scarecrow Press.

FRANCESChINA, JoHn ET AL.

2017 Incidental and Dance Music in the American Theatre from 1786 to 1923: Volume 3. Georgia: BearManor Media (eBook).

Giro, RADAMÉs

2007 Diccionario Enciclopédico de la Música en Cuba, vol. 4, La Habana: Editorial Letras Cubanas.

GOLDBERG, BETHANY

2013 "Matzka, George", Grove Music Online. www.oxfordmusiconline.com/subscriber/article/.grove/ music/A2084006 [acceso: 8 de diciembre, 2012].

GotTSCHalk, Louis Moreau

2006 Notes of a Pianist. Editado por Jeanne Behrend. Princeton/Oxford: Princeton University.

GuZMÁn, JuAn PÉREZ de

1876 "Esmeralda Cervántes", La Ilustración Española y Americana (Madrid), XX/44 (30 de noviembre), pp. 333-338, www.bnc.cat/esl/Visitanos/Exposiciones/Esmeralda-Cervantes-larestauracio-del-seu-Album [acceso: 9 de enero, 2018].

Haws, Barbara

2013 "New York Philharmonic", Grove Music Online. www.oxfordmusiconline.com/grovemusic/ view/10.1093/gmo/.9781561592630.001.0001/omo-9781561592630-e-1002252043 [acceso: 20 de junio, 2018].

Hines, Dixie y Harry Prescott Hanaford (editores)

1914 Who's Who in Music and Drama, An Encyclopaedia of Biography of Notable Men and Women in Music and the Drama, Nueva York: H. P. Hanaford. 
Hoover, Cynthia Adams

2001 "Steinway", Grove Music Online. www.oxfordmusiconline.com/grovemusic/view/10.1093/ gmo/.9781561592630.001.0001/o_mo-9781561592630-e-0000026663 [acceso: 28 de junio, 2018].

Krehbiel, Henry EdWARd

1892 The Philharmonic Society: A Memorial. Nueva York: Novello, Ewer.

LAPIQUE, ZoILA

2008 Cuba Colonial: Música, compositores e intérpretes (1570-1902). La Habana: Letras Cubanas.

LiEDERKRANZ NeW YorK

2018 "History", Liederkranz New York, www.liederkranzny.org/history.html [acceso: 29 de mayo, 2018].

LotT, Allen R.

2003 From Paris to Peoria: How European Piano Virtuosos Brought Classical Music to the American Heartland. Nueva York: Oxford University Press.

MCCABE, James D.

1876 The Illustrated History of the Centennial Exhibition. Filadelfia, PA: The National Publishing Co.

Martínez-Alier, Verena

1989 Marriage, Class and Color in Nineteenth-Century Cuba. Ann Arbor: University of Michigan Press.

Merino Montero, Luis

1990 Repercusiones nacionales e internacionales de la visita a Chile de José White. Revista Musical Chilena, XLIV/173 (enero-junio), pp. 65-113.

Milanca Guzmán, Mario

1990a "Dos cartas inéditas: Teresa Carreño le escribe a José White”, Boletín de la Academia Nacional de la Historia, V. LXXIII, No. 289, (enero-marzo), Caracas, pp. 69-83.

1990b "José White en Venezuela”, Revista Musical Chilena, XLIV/173 (enero-junio), pp. 25-64.

2002 “6. Carreño García de Sena, Teresa”, Diccionario de la Música Española e Hispanoamericana. Editado por Emilio Casares Rodicio. Tomo II. Madrid: S.G.A.E., pp. 234-237.

Morrisey, James W.

1904 Noted men and women; a profusely illustrated book, containing the humor, wit, sentiment and diplomacy in the social, artistic and business lives of people herein set fourth. Nueva York: Klebold Press.

Nelson-Strauss, Brenda y John Spitzer (EDitores)

2012 "Theodore Thomas and the Cultivation of American Music", American Orchestras in the Nineteen Century, Chicago/Londres: University of Chicago Press, pp. 395-434.

Petrucci Music Library

2016 "List of Works by Henri Vieuxtemps", Petrucci Music Library, http://imslp.org/wiki/List_of_ works_by_Henri_Vieuxtemps [acceso: 27 de junio, 2018].

Pierre, Constant

1900 Le Conservatoire national de musique et de déclamation: documents historiques et administratifs. París: Imprimerie nationale.

Pisani, Michael V.

2014 Music for the Melodramatic Theatre in Nineteenth-Century London and New York. Iowa: University of Iowa Press.

Preston, Katherine K.

2017 Opera for the People, English-Language Opera and Women Managers in Late 19th-Century America. Nueva York: Oxford University Press.

RADOUX, JEAN THÉOdore

1891 Vieuxtemps: Sa vie, ses æeuvres. Lieja: A. Bénard. 
Reichert, MatThew

2011 "Carl Bergmann in New York: Conducting Activity 1852-1876”, DMA Diss., The City University of New York.

Russell, Charles EdWARd

1927 The American Orchestra and Theodore Thomas. Garden City, Nueva York: Doubleday, Page \& Company.

SAFFle, Michael

1998 Music and Culture in America, 1861-1918, Londres: Routledge.

SCHWARZ, Boris, AND ZOFIA CHECHLIŃSKa

2001 “(1) Henryk [Henri] Wieniawski”, Grove Music Online. www.oxfordmusiconline.com/grovemusic/ view/10.1093/gmo/.978156.1592630.001.0001/omo-9781561592630-e-0000030284 [acceso: 28 de junio, 2018].

SHANET, HoWARD

1975 Philharmonic: A History of New York's Orchestra. Garden City, Nueva York. Doubleday \& Company Inc.

SLONIMSKY, Nicholas

1958 “Steinway \& Sons”, Baker's Biographical Dictionary of Musicians, Nueva York: Schirmer, 1958, p. 1566.

SPITZER, JOHN (EDITOR)

2012 "Orchestral Repertory: Highbrow and Lowbrow", American Orchestras in the Nineteen Century, Chicago/Londres: University of Chicago Press, pp. 367-371.

STEINWAY HaLL

2016 "Steinway and Sons History", Steinway E Sons, Steinway Hall Official Webpage, http://steinway. co.uk/about-us/ [acceso: 28 de junio, 2018].

Stevenson, Robert M.

1983 “Carreño’s 1875 California Appearances”, Inter-American Music Review, 10 (primavera-verano), pp. $9-15$.

2004 "Teresa Carreño (1853-1917): Remembered on her 150th Anniversary", Latin American Music Review, 25/2 (otoño-invierno), pp. 163-179.

The Library Company

2008 The Library Company website, www.lcpimages.org/centennial/ [acceso: 25 de junio, 2018].

Thompson, Michael

2010 “Corliss Centennial Engine”, The New England Wireless and Steam Museum, http://newsm.org/ steam-e/corliss-centennial-engine/ [acceso: 25 de junio, 2018].

Toledo, Armando

1990 "White en Cuba", Revista Musical Chilena 44/173 (enero-junio), pp. 5-24.

Trotter, James M.

1878 Music and Some Highly Musical People. Boston: Lee and Shepard Publishers, Nueva York: Charles T. Dillingham.

Trujillo, Iraida ET AL.

2005 José White. Matanzas: Ediciones Vigía.

Upton, GEORGE P. (EDitor)

1905 Theodore Thomas: A Musical Autobiography, Concert Programmes, vol. II. Chicago: McClurg.

\section{PERIÓDICOS DEL SIGLO XIX}

- $\quad$ Boston Post (1876)

- Dwight's Journal of Music, Boston (1876) 
- La Ilustración Española y Americana, Madrid (1876)

- New Orleans Republican (1875)

- New York Clipper (1876)

- $\quad$ New York Herald $(1875,1876)$

- $\quad$ New-York Tribune $(1875,1876)$

- $\quad$ Staten Island NY Evening Post (1876)

- $\quad$ The Boston Daily Globe (1876)

- $\quad$ The Brooklyn Daily Eagle $(1875,1876)$

- The Musician and Artist, Boston (1876)

- $\quad$ The New York Times $(1875,1876)$

- $\quad$ The Times, Philadelphia (1876)

- $\quad$ The York Daily, Pennsylvania (1875)

\section{MATERIALES DE ARCHIVO}

- Archives Center, National Museum of American History, Smithsonian Institution, William Steinway's Diary (20 April 1861 to 8 November 1896).

- Archives Center, National Museum of American History, Smithsonian Institution, The Jacques Français Rare Violins Inc. Photographic Archive and Business Records, The sales ledgers, 1845-1938, Box 55, Folders 2 and 4. (Nicholas Sackman translator, 2018).

- Archives départementales de Seine-Maritime, France, Steamship "Le Pereire", passengers Mme. White and maid. Le Havre-New York. 6P6/393, Roll 166, view 101. Departure: December 4, 1875. Archive Département Seine-Maritime, France.

- Archives départementales de Seine-Maritime, France, Steamship "Amérique," passenger Mme. White. New York-Le Havre. 6P6/407, Roll ¿?, view 128. Departed: February 19. Arrived March 1, 1876. Archive Département Seine-Maritime, France.

- Brooklyn Historical Society, Clementine Lasar Studwell Scrapbook.

- Historical Society of Pennsylvania Archives Department.

- New York Philharmonic, Leon Levy Digital Archives, Secretary's Accounts Income and Expenses Ledger 1868-1885, May 1, 1868-April 16, 1885 [ID: 498-01-15].

- New York Philharmonic, Leon Levy Digital Archives, Meeting of Friday, December 3, 1875, at the Academy of Music. Board of Directors Meeting Minutes, 1874-1877, June 6, 1871-September 28, 1877 [ID: 498-01-07]).

- New York Philharmonic, Leon Levy Digital Archives, Meeting of December 17, 1875 and March 24, 1876. Business Meeting Minutes, 1866-1882, June 2, 1866-April 25, 1882 [ID: 498-01-09].

- New York Philharmonic, Leon Levy Digital Archives, Meeting of March 25, 1876. Board of Directors Meeting Minutes, 1874-1877, June 6, 1871-September 28, 1877 [ID: 498-01-07]).

- United States Customs Service, National Archives, NY Record of Arrivals. Steamship "Pereira," passengers Mme. White and Maid. Havre to New York. Arrival: December 16. M237,1820-1897, Roll 400, View 1340. Records of the U.S. Customs Service, National Archives.

\section{COMUNICACIÓN POR CORREO ELECTRÓNICO}

Gabryel Smith, Assistant Archivist, New York Philharmonic (11 de enero y 4 de julio, 2018). 